Open Access

\title{
Synthesis, radiosynthesis, in vitro and first in vivo evaluation of a new matrix metalloproteinase inhibitor based on $\gamma^{-}$ fluorinated a-sulfonylaminohydroxamic acid
}

Verena Hugenberg ${ }^{1,2,6^{*}}$, Malte Behrends ${ }^{3}$, Stefan Wagner ${ }^{2}$, Sven Hermann ${ }^{1,4}$, Michael Schäfers ${ }^{1,2,4}$, Hartmuth C. Kolb ${ }^{5}$, Katrin Szardenings ${ }^{5}$, Joseph C. Walsh ${ }^{5}$, Luis F. Gomez ${ }^{5}$, Klaus Kopka ${ }^{2,7}$ and Günter Haufe , $^{1,4^{*}}$ (i)

\author{
* Correspondence: vhugenberg@ \\ hdz-nrw.de; haufe@uni-muenster.de \\ 'European Institute for Molecular \\ Imaging, University of Münster, \\ Waldeyerstr. 15, D-48149 Münster, \\ Germany \\ Full list of author information is \\ available at the end of the article
}

\begin{abstract}
Background: To study MMP activity in vivo in disease, several radiolabeled MMP inhibitors functioning as radiotracers have been evaluated by means of SPECT and PET. Unfortunately, most of them suffer from metabolic instability, mainly hepatobiliary clearance and insufficient target binding. The introduction of a fluorine atom into MMPIs could contribute to target binding, enhance the metabolic stability and might shift the clearance towards more renal elimination. Recently developed asulfonylaminohydroxamic acid based $y$-fluorinated inhibitors of MMP-2 and -9 provide promising fluorine interactions with the enzyme active site and high MMP inhibition potencies. The aim of this study is the (radio)synthesis of a $\gamma$-fluorinated MMP-2 and -9 inhibitor to evaluate its potential as a radiotracer to image MMP activity in vivo.

Results: Two new fluorine-containing, enantiomerically pure inhibitors for MMP-2 and -9 were synthesized in a six step sequence. Both enantiomers exhibited equal inhibition potencies in the low nanomolar and subnanomolar range. $\log D$ value indicated better water solubility compared to the CGS 25966 based analog. The most potent inhibitor was successfully radiofluorinated. In vivo biodistribution in wild type mice revealed predominantly hepatobiliary clearance. Two major radioactive metabolites were found in different organs. Defluorination of the radiotracer was not observed.

Conclusion: (Radio)synthesis of a CGS based $y$-fluorinated MMP inhibitor was successfully accomplished. The (S)-enantiomer, which normally shows no biological activity, also exhibited high MMP inhibition potencies, which may be attributed to additional interactions of fluorine with enzyme's active site. Despite higher hydrophilicity no significant differences in the clearance characteristics compared to non-fluorinated MMPIs was observed. Metabolically stabilizing effect of the fluorine was not monitored in vivo in wild type mice.
\end{abstract}

Keywords: Matrix metalloproteinase inhibitors, CGS 27023A analogues, In vitro assay, Amino hydroxamic acid, Fluorine; radiotracer, In vivo biodistribution 


\section{Background}

Matrix metalloproteinases (MMPs) are a family of structurally and functionally related zinc and calcium-dependent endopeptidases, responsible for the degradation and reconstruction of protein components within the extracellular matrix (ECM). They are involved in many physiological as well as pathological processes (Whittaker et al. 1999; Woessner and Nagase, 2000). Upregulated levels of MMPs are associated with various pathologies like inflammation, atherosclerosis (Brauer. 2006; Rai and Agrawal, 2017), and tumor progression (Sternlicht. 2001; Fingleton. 2006; Kessenbrock et al. 2010). MMP-2 and MMP-9 for example are capable to degrade type IV collagen, the most abundant component of the basement membrane. Degradation of the basement membrane allows cancer cells to migrate out of the primary tumor to form metastases. Upregulated expression of the gelatinases MMP-2 and MMP-9 directly correlates to an increased proliferation of tumor cells and tumor growth (Zhong et al. 2018). Especially for the diagnosis of breast cancer (Ren et al. 2015), non-small lung cancer (Gong et al. 2016) and ovarian endothelial carcinoma (Jia et al. 2017) the overexpression of MMP-2 and/or MMP-9 is used as a prognostic biomarker. Elevated levels of MMP-9 are found in rheumatoid arthritis (Gruber et al. 1996) and correlate with the progression of idiopathic atrial fibrillation ( $\mathrm{Li}$ et al. 2014). Furthermore, overexpression of MMP-2 and -9 play a significant role in cardiovascular disease (Dimas et al. 2017) and in the development of aortic aneurysms (Rabkin. 2017). Therefore, many different MMP inhibitors (MMPIs) of the activated enzymes have been developed in recent years (Levin et al. 2017). Most of these MMPIs are broad spectrum inhibitors and gain their potency via the direct interaction with the catalytic $\mathrm{Zn}(\mathrm{II})$ ion in the active center of the enzyme (Jacobsen et al. 2010). Among different synthetic MMPIs, the sulfonamide-based hydroxamic acid derivatives CGS 25966 and CGS 27023A (Fig. 1) have been intensively investigated. Interestingly only the $(R)$-enantiomers of these hydroxamic acid derivatives are potent MMP inhibitors (Table 1), while the $(S)$-enantiomeric form demonstrated low or no inhibition potencies (Mac Pherson et al. 1997; Scozzafava et al. 1997) To the best of our knowledge, no $\mathrm{IC}_{50}$ values were published for the $(S)$-enantiomers.

Based on lead structure CGS 27030A our group recently developed new potent fluorinated inhibitors 1 and 2 of MMP-2 and -9 (Table 1) (Behrends et al. 2015). Bearing a fluorine substituent in the $\gamma$-position of the amino acid core, these compounds seem to provide an additional possibility for the interaction with the active site of MMP-2 and MMP-9 and thereby, reducing the need of the stereocenter. In contrast to the lead compound CGS 27030A, where only the $(R)$-enantiomer shows inhibition potency for MMP-2 and -9, both enantiomers of the fluorinated hydroxamates 1 and $\mathbf{2}$ are potent

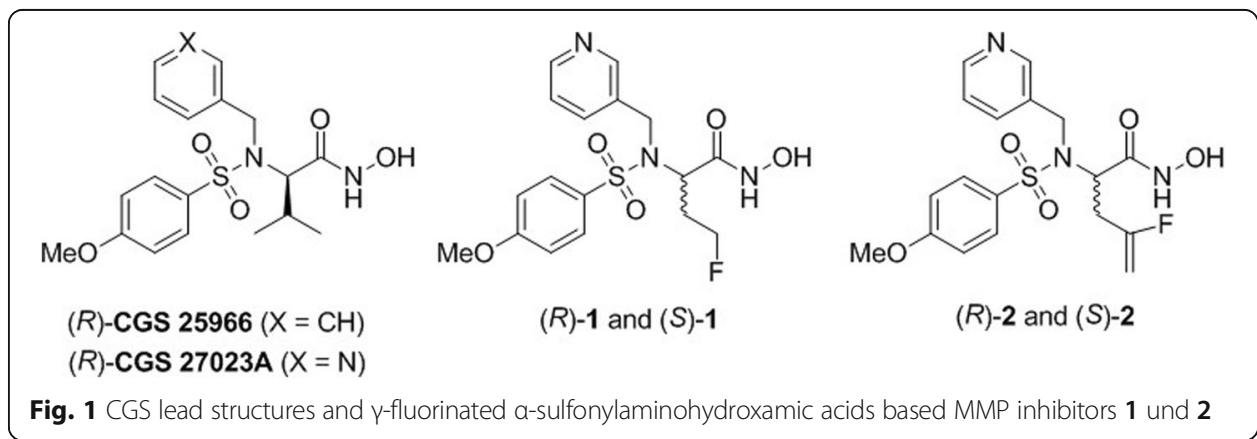


Table 1 IC $C_{50}$ vcalues of CGS lead structures and $\gamma$-fluorinated MMP inhibitors 1 und 2 (Mac Pherson et al. 1997; Scozzafava et al. 2000; Behrends et al. 2015)

\begin{tabular}{llll}
\hline Compound & Configuration & \multicolumn{2}{l}{$\boldsymbol{C}_{50}$ values [nM] } \\
\cline { 3 - 4 } & & MMP-2 & MMP-9 \\
\hline CGS 27023A & $R$ & 20 & 8 \\
CGS 25966 & $R$ & 11 & 27 \\
$\mathbf{1}$ & $R$ & 6.4 & 12.3 \\
& $S$ & 32.8 & 3.0 \\
$\mathbf{2}$ & $R$ & 9.3 & 8.3 \\
& S & 7.2 & 4.9 \\
\hline
\end{tabular}

inhibitors (Fig. 1). Modeling studies suggest that the $\gamma$-fluorine atom in the core of $\alpha$-aminosulfonyl hydroxamic acid residues appear to influence the relative potencies via specific inhibitor-peptidase interactions, including short fluorine-hydrogen contacts, within the enzyme's subpockets (Behrends et al. 2015).

To study MMP activity in vivo in disease, radiolabeled MMPIs functioning as radiotracers have been synthesized and evaluated by means of SPECT and PET. Unfortunately, most of the radiolabeled MMPIs suffer from insufficient metabolic instability, fast excretory elimination and insufficient target binding. Characteristically, the fast blood clearance and therefore insufficient bioavailability of radiolabeled MMPIs is based on a very efficient hepatobiliary tracer elimination (Matusiak et al. 2013).

The introduction of fluorine atom(s) into organic molecules is known to influence their pharmacokinetics, binding affinities and lipophilicity (O'Hagan. 2008; Purser et al. 2008; Begué and Bonnet-Delpon, 2008; Yamazaki et al. 2009; Gillis et al. 2015; Huchet et al. 2015; Meanwell, 2018). Thus, linkage of an additional fluorine-containing functional group in remote position to the zinc binding moiety might contribute to more efficient binding of the inhibitor in the active center of the enzyme (Behrends et al. 2015). Moreover, it could enhance the metabolic stability and might retard hepatobiliary elimination resulting in a shift of the clearance towards more renal elimination due to changes in hydrophilicity. In addition, a radiolabeled $\gamma$-fluorinated $\alpha$-aminohydroxamic acid could possibly represent an effective radiotracer for imaging MMP-2 and -9 with improved pharmacokinetic properties and overall target binding affinities. Hence, the aim of this work is the synthesis of a $\gamma$-fluorinated MMP-2 and -9 inhibitor based on $\alpha$-sulfonylaminohydroxamic acid with an additional fluorine-containing substituent allowing ${ }^{18} \mathrm{~F}$-radiolabeling, and evaluation of its potential for the application as a radiotracer to image MMP activity in vivo.

\section{Methods}

General methods and chemistry

All chemicals, reagents and solvents for the synthesis of the compounds were analytical grade, purchased from commercial sources and used without further purification unless otherwise specified. All air and moisture-sensitive reactions were performed under argon atmosphere. Solvents were purified and dried analog to literature methods, where necessary. The melting points (mp) are uncorrected and were determined in capillary tubes on a Stuart Scientific SMP3 capillary melting point apparatus. Column 
chromatography was performed on Merck silica gel $60(0.040-0.063 \mathrm{~mm})$. Thin layer chromatography (TLC) was carried out on silica gel-coated polyester backed TLC plates (Polygram, SIL G/UV $\mathrm{UV}_{254}$, Macherey \& Nagel) using solvent mixtures of cyclohexane $(\mathrm{CH})$, ethyl acetate (EA) and methanol $(\mathrm{MeOH})$. Compounds were visualized by UV light $(254 \mathrm{~nm})$. NMR spectra were recorded in $\mathrm{CDCl}_{3}, \mathrm{CD}_{3} \mathrm{OH}$ or DMSO- $d_{6}$ on a Bruker ARX300, a Bruker DPX300 $\left({ }^{1} \mathrm{H}\right.$ NMR, $300 \mathrm{MHz},{ }^{13} \mathrm{C}$ NMR, $75 \mathrm{MHz},{ }^{19} \mathrm{~F}$ NMR, $282 \mathrm{MHz})$, a Bruker AMX $400\left({ }^{1} \mathrm{H} \mathrm{NMR}, 400 \mathrm{MHz},{ }^{13} \mathrm{C} \mathrm{NMR}, 100 \mathrm{MHz}\right)$ and a Varian Unity plus $600\left({ }^{1} \mathrm{H}\right.$ NMR, $600 \mathrm{MHz},{ }^{13} \mathrm{C}$ NMR, $\left.151 \mathrm{MHz}\right)$ spectrometer. TMS $\left({ }^{1} \mathrm{H}\right)$, $\mathrm{CDCl}_{3}$, DMSO- $d_{6}, \mathrm{CD}_{3} \mathrm{OD}\left({ }^{13} \mathrm{C}\right)$ and $\mathrm{CFCl}_{3}\left({ }^{19} \mathrm{~F}\right)$ were used as internal standards and all chemical shift values are reported in ppm $(\delta)$. Exact mass analyses were conducted on a Bruker MicroTof apparatus. The chemical and radiochemical purities of each new non-radioactive and radioactive compound were $\geq 95 \%$ and assessed by analytical gradient reversed-phase HPLC system A. HPLC system A: Two K-1800 pumps and an S-2500 UV detector (Herbert Knauer GmbH), a GabiStar $\gamma$-detector (Raytest Isotopenmessgeräte $\mathrm{GmbH}$ ). The recorded data were processed by the ChromGate HPLC software (Herbert Knauer GmbH). The HPLC method A1 started with a linear gradient from $10 \%$ to $90 \% \mathrm{CH}_{3} \mathrm{CN}$ in water (0.1\% TFA) over $9 \mathrm{~min}$, followed by a linear gradient from $90 \%$ to $10 \% \mathrm{CH}_{3} \mathrm{CN}$ in water $(0.1 \%$ TFA) over $6 \mathrm{~min}$, with a flow rate of $1 \mathrm{~mL} \cdot \mathrm{min}^{-1}$.

p-(2-Fluoroethoxy)phenylsulfonyl chloride (3a) (Wagner et al. 2011), 2-[4-(chlorosulfonyl)phenoxy] ethyl-4-methylbenzenesulfonate (3b) (Wagner et al. 2011), tert-butyl (S)-2-amino-4-fluoropent-4-enecarboxylate $((S)$-4) (Laue et al. 2000) and tert-butyl (R)-2-amino-4-fluoropent-4-enecarboxylate ((S)-4) (Laue et al. 2000) were synthesized following literature procedures. All animal experiments were conducted in accordance with local institutional guidelines for the care and use of laboratory animals.

\section{Preparation of MMPI (S)-9a}

Tert-butyl (S)-4-fluoro-2-\{[4-(2-fluoroethoxy)phenyl]sulfonamide\}pent-4-enoate ((S)-5a)

To a solution of tert-butyl (S)-2-amino-4-fluoropent-4-enecarboxylate ((S)-4) (892 mg, $4.7 \mathrm{mmol}$ ) in pyridine $p$-(2-fluoroethoxy)-phenylsulfonylchloride $(1130 \mathrm{mg}, 4.7 \mathrm{mmol}$ ) was added under stirring at $0{ }^{\circ} \mathrm{C}$. The reaction mixture was allowed to warm up to rt. and stirred for $40 \mathrm{~h}$. Then the mixture was diluted with dichloromethane and the organic solution was washed with $0.5 \mathrm{~N} \mathrm{HCl}$, water (2 times each) and brine. After drying with magnesium sulfate the solvent was removed in vacuum. The crude product was passed through a short pad of silica gel and recrystallized from ethyl acetate/cyclohexane. The product was isolated as a colorless crystalline solid $(1.18 \mathrm{~g}, 64 \%)$; mp $102{ }^{\circ} \mathrm{C}(\mathrm{EA} / \mathrm{CH}) .{ }^{1} \mathrm{H}$ $\operatorname{NMR}\left(300 \mathrm{MHz}, \mathrm{CDCl}_{3}\right): \delta=7.79\left(\mathrm{dm},{ }^{3} \mathrm{~J}_{\mathrm{H}, \mathrm{H}}=9.0 \mathrm{~Hz}, \mathrm{Ar} H, 2 \mathrm{H},\right), 6.99\left(\mathrm{dm},{ }^{3} J_{\mathrm{H}, \mathrm{H}}=\right.$ $9.0 \mathrm{~Hz}, \mathrm{ArH}, 2 \mathrm{H}), 5.37\left(\mathrm{~d},{ }^{3} J_{\mathrm{H}, \mathrm{H}}=9.0 \mathrm{~Hz}, \mathrm{NH}, 1 \mathrm{H}\right), 4.77\left(\mathrm{dm},{ }^{2} J_{\mathrm{H}, \mathrm{F}}=47.5 \mathrm{~Hz}, \mathrm{CH}_{2} \mathrm{~F}, 2 \mathrm{H}\right)$, $4.64\left(\mathrm{dd},{ }^{3} J_{\mathrm{H}, \mathrm{F}}=17.1 \mathrm{~Hz},{ }^{2} J_{\mathrm{H}, \mathrm{H}}=3.1 \mathrm{~Hz}, \mathrm{CF}=\mathrm{CH}_{2}, \mathrm{H}_{c i s}, 1 \mathrm{H}\right), 4.35\left(\mathrm{dd},{ }^{3} J_{\mathrm{H}, \mathrm{F}}=49.3 \mathrm{~Hz},{ }^{2} J_{\mathrm{H}, \mathrm{H}}\right.$ $\left.=3.1 \mathrm{~Hz}, \mathrm{CF}=\mathrm{CH}_{2}, \mathrm{H}_{\text {trans }}, 1 \mathrm{H}\right), 4.26\left(\mathrm{dm},{ }^{3} \mathrm{~J}_{\mathrm{H}, \mathrm{F}}=27.7 \mathrm{~Hz}, \mathrm{CH}_{2} \mathrm{CH}_{2} \mathrm{~F}, 2 \mathrm{H}\right), 3.98\left(\mathrm{dt},{ }^{3} J_{\mathrm{H}, \mathrm{H}}=\right.$ $\left.8.8 \mathrm{~Hz},{ }^{3} J_{\mathrm{H}, \mathrm{H}}=5.8 \mathrm{~Hz}, \mathrm{NHCH}, 1 \mathrm{H}\right), 2.65\left(\mathrm{~d},{ }^{3} J_{\mathrm{H}, \mathrm{H}}=5.9 \mathrm{~Hz}, \mathrm{NHCHCH}_{2}, \mathrm{H}_{A}, 1 \mathrm{H}\right), 2.59(\mathrm{dd}$, $\left.{ }^{3} J_{\mathrm{H}, \mathrm{H}}=5.7 \mathrm{~Hz},{ }^{3} J_{\mathrm{H}, \mathrm{H}}=4.2 \mathrm{~Hz}, \mathrm{NHCHCH}_{2}, \mathrm{H}_{\mathrm{B}}, 1 \mathrm{H}\right), 1.30\left(\mathrm{~s}, \mathrm{C}\left(\mathrm{CH}_{3}\right)_{3}, 9 \mathrm{H}\right) .{ }^{13} \mathrm{C} \mathrm{NMR}$ (75 MHz, $\left.\mathrm{CDCl}_{3}\right): \delta=169.2(\mathrm{CO}), 161.8(\mathrm{ArCO}), 160.9$ (d, $\left.{ }^{1} J_{\mathrm{C}, \mathrm{F}}=256.9 \mathrm{~Hz}, \mathrm{CF}\right), 131.9$ $\left(\mathrm{ArCSO}_{2}\right), 129.5(\mathrm{ArCH}), 114.7(\mathrm{ArCH}), 94.1\left(\mathrm{~d},{ }^{2} J_{\mathrm{C}, \mathrm{F}}=18.8 \mathrm{~Hz}, \mathrm{CH}_{2}=\mathrm{CF}\right), 83.2$ $\left(C\left(\mathrm{CH}_{3}\right)_{3}\right), 81.5\left(\mathrm{~d},{ }^{1} J_{\mathrm{C}, \mathrm{F}}=171.5 \mathrm{~Hz}, \mathrm{CH}_{2} \mathrm{~F}\right), 67.4\left(\mathrm{dt},{ }^{2} J_{\mathrm{C}, \mathrm{F}}=20.4 \mathrm{~Hz}, \mathrm{CH}_{2} \mathrm{CH}_{2} \mathrm{~F}\right), 53.2$ 
$\left(\mathrm{d},{ }^{3} J_{\mathrm{C}, \mathrm{F}}=1.0 \mathrm{~Hz}, \mathrm{NHCH}\right), 36.4\left(\mathrm{dt},{ }^{2} J_{\mathrm{C}, \mathrm{F}}=27.6 \mathrm{~Hz}, \mathrm{NHCHCH} 2\right), 27.6\left(\mathrm{C}\left(\mathrm{CH}_{3}\right)_{3}\right) .{ }^{19} \mathrm{~F} \mathrm{NMR}$ $\left(282 \mathrm{MHz}, \mathrm{CDCl}_{3}\right): \delta=-96.1\left(\mathrm{ddt},{ }^{3} J_{\mathrm{H}, \mathrm{F}}=49.3 \mathrm{~Hz},{ }^{3} J_{\mathrm{H}, \mathrm{F}}=20.4 \mathrm{~Hz},{ }^{3} J_{\mathrm{H}, \mathrm{F}}=17.2 \mathrm{~Hz}, \mathrm{CF}\right),-$ $224.3\left(\mathrm{tt},{ }^{2} \mathrm{~J}_{\mathrm{H}, \mathrm{F}}=47.3 \mathrm{~Hz},{ }^{3} \mathrm{~J}_{\mathrm{H}, \mathrm{F}}=27.7 \mathrm{~Hz}, \mathrm{CH}_{2} \mathrm{~F}\right)$. Elemental analysis: $\mathrm{C}_{17} \mathrm{H}_{23} \mathrm{~F}_{2} \mathrm{NO}_{5} \mathrm{~S}(\mathrm{M}=$ $391.43 \mathrm{~g} / \mathrm{mol}$ ), calcd. $\mathrm{C}$ 52.16, H 5.92, N 3.58; found $\mathrm{C}$ 52.64, $\mathrm{H}$ 6.24, N 3.55\%. MS-ES(+)-EM: $m / z=414.1160\left[(\mathrm{M}+\mathrm{Na})^{+}\right]$calcd. For $\mathrm{C}_{17} \mathrm{H}_{23} \mathrm{~F}_{2} \mathrm{NO}_{5} \mathrm{SNa}^{+}: 414.1163$.

Tert-butyl (S)-2-\{[N-benzyl-4-(2-fluoroethoxy)phenyl]sulfonamide\}-4-fluoropent-4-enoate ((S)-6a) Tert-Butyl $N$-[p-(-2-fluoroethoxy)phenylsulfonyl]aminopent-4-enoate $(830 \mathrm{mg}, 2.12 \mathrm{mmol})$, dissolved in dimethylformamide $(20 \mathrm{~mL})$ was treated under stirring with 10 equivalents of potassium carbonate $(2.93 \mathrm{~g}, 21.2 \mathrm{mmol}$ ). After $20 \mathrm{~min}$ benzyl bromide (363 mg, $2.12 \mathrm{mmol}$, 1 equiv) was added and the mixture was stirred at rt. for $40 \mathrm{~h}$. Then water was added and the mixture was extracted with ethyl acetate (4 times). The combined organic extracts were washed with water (4 times), brine and dried with magnesium sulfate. After removal of the solvent the crude product was purified chromatographically (silica gel, CH/EA, 4:1). The product was obtained as a colorless yellowish viscos oil (800 mg, 78\%). ${ }^{1} \mathrm{H}$ NMR (300 MHz, $\left.\mathrm{CDCl}_{3}\right): \delta=7.82\left(\mathrm{dm},{ }^{3} J_{\mathrm{H}, \mathrm{H}}=9.0 \mathrm{~Hz}, \mathrm{ArH}, 2 \mathrm{H}\right), 7.29(\mathrm{~m}, 14-\mathrm{CH}, 15-\mathrm{CH}, \mathrm{ArH}, 5 \mathrm{H}), 6.98$ $\left(\mathrm{dm},{ }^{3} J_{\mathrm{H}, \mathrm{H}}=8.9 \mathrm{~Hz}, \mathrm{ArH}, 2 \mathrm{H}\right), 4.80\left(\mathrm{dm},{ }^{2} J_{\mathrm{H}, \mathrm{F}}=47.3 \mathrm{~Hz}, \mathrm{CH}_{2} \mathrm{~F}, 2 \mathrm{H}\right), 4.65\left(\mathrm{~d},{ }^{2} J_{\mathrm{H}, \mathrm{H}}=16.0 \mathrm{~Hz}\right.$, $\left.\mathrm{NCH}_{2}, \mathrm{H}_{\mathrm{A}}, 1 \mathrm{H}\right), 4.56\left(\mathrm{t},{ }^{3} J_{\mathrm{H}, \mathrm{H}}=7.3 \mathrm{~Hz}, \mathrm{NCH}, 1 \mathrm{H}\right), 4.53\left(\mathrm{dd},{ }^{3} J_{\mathrm{H}, \mathrm{F}}=17.1 \mathrm{~Hz},{ }^{2} J_{\mathrm{H}, \mathrm{H}}=3.0 \mathrm{~Hz}\right.$, $\left.\mathrm{CF}=\mathrm{CH}_{2}, \mathrm{H}_{\text {cis }}, 1 \mathrm{H}\right), 4.29\left(\mathrm{~d},{ }^{2} J_{\mathrm{H}, \mathrm{H}}=15.7 \mathrm{~Hz}, \mathrm{NCH}_{2}, \mathrm{H}_{\mathrm{B}}, 2 \mathrm{H}\right), 4.25\left(\mathrm{dm},{ }^{3} J_{\mathrm{H}, \mathrm{F}}=27.8 \mathrm{~Hz}\right.$, $\left.\mathrm{CH}_{2} \mathrm{CH}_{2} \mathrm{~F}, 2 \mathrm{H}\right), 4.11\left(\mathrm{dd},{ }^{3} \mathrm{~J}_{\mathrm{H}, \mathrm{F}}=49.9 \mathrm{~Hz},{ }^{2} J_{\mathrm{H}, \mathrm{H}}=3.0 \mathrm{~Hz}, \mathrm{CF}=\mathrm{CH}_{2}, \mathrm{H}_{\text {trans }}, 1 \mathrm{H}\right), 2.70\left(\mathrm{dt},{ }^{3} J_{\mathrm{H}, \mathrm{F}}\right.$ $\left.=15.3 \mathrm{~Hz},{ }^{3} J_{\mathrm{H}, \mathrm{H}}=6.9 \mathrm{~Hz}, \mathrm{NCH}_{2}, \mathrm{H}_{\mathrm{A}}, 1 \mathrm{H}\right), 2.46\left(\mathrm{ddd},{ }^{3} J_{\mathrm{H}, \mathrm{F}}=20.0 \mathrm{~Hz},{ }^{2} J_{\mathrm{H}, \mathrm{H}}=15.1 \mathrm{~Hz},{ }^{3} J_{\mathrm{H}, \mathrm{H}}=\right.$ $\left.7.6 \mathrm{~Hz}, \mathrm{NCH}_{2}, \mathrm{H}_{\mathrm{B}}, 1 \mathrm{H}\right), 1.36\left(\mathrm{~s}, \mathrm{C}\left(\mathrm{CH}_{3}\right)_{3}, 9 \mathrm{H}\right) .{ }^{13} \mathrm{C} \mathrm{NMR}\left(75 \mathrm{MHz}, \mathrm{CDCl}_{3}\right): \delta=168.5(\mathrm{CO})$, $161.9\left(\mathrm{ds},{ }^{1} J_{\mathrm{C}, \mathrm{F}}=256.5 \mathrm{~Hz}, \mathrm{CF}\right), 161.7(\mathrm{ArCO}), 136.7\left(\mathrm{ArCCH}_{2}\right), 132.5\left(\mathrm{ArCSO}_{2}\right), 129.9$ $(\mathrm{ArCH}), 128.5(\mathrm{ArCH}), 128.4(\mathrm{ArCH}), 127.7(\mathrm{ArCH}), 114.6(\mathrm{ArCH}), 93.1\left(\mathrm{dt},{ }^{2} J_{\mathrm{C}, \mathrm{F}}=19.2 \mathrm{~Hz}\right.$,

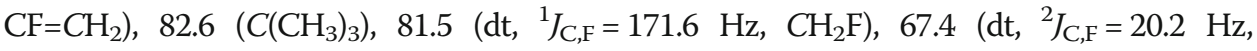

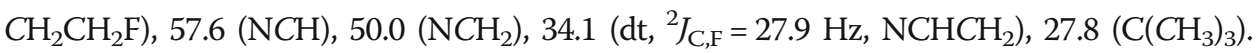
${ }^{19} \mathrm{~F}$ NMR $\left(282 \mathrm{MHz}, \mathrm{CD}_{3} \mathrm{CN}\right): \delta=-97.7\left(\mathrm{~m},{ }^{3} J_{\mathrm{H}, \mathrm{F}}=49.8 \mathrm{~Hz},{ }^{3} J_{\mathrm{H}, \mathrm{F}}=19.8 \mathrm{~Hz},{ }^{3} J_{\mathrm{H}, \mathrm{F}}=\right.$ 16.4.0 Hz, CF), $-224.3\left(\mathrm{tt}^{2}{ }^{2} \mathrm{H}_{\mathrm{H}, \mathrm{F}}=47.3 \mathrm{~Hz},{ }^{3} J_{\mathrm{H}, \mathrm{F}}=27.6 \mathrm{~Hz}, \mathrm{CH}_{2} \mathrm{~F}\right) . \mathrm{MS}-\mathrm{ES}(+)-\mathrm{EM}: m / z$ $=504.1613\left[(\mathrm{M}+\mathrm{Na})^{+}\right]$calcd. For $\mathrm{C}_{24} \mathrm{H}_{29} \mathrm{~F}_{2} \mathrm{NO}_{5} \mathrm{SNa}^{+}: 504.1632$.

\section{(S)-2-\{[N-Benzyl-4-(2-fluoroethoxy)phenyl]sulfonamide\}-4-fluoropent-4-enoic acid ((S)-7a)}

In a dried YOUNG-tube tert-butyl $N$-benzyl-(S)- $N$-[ $p$-(2-fluoroethoxy)phenyl-sulfony1]-2-amino-4-fluoropent-4-enoate ((S)-6a) (400 mg, $0.94 \mathrm{mmol}$ ) was dissolved in dry dichloromethane $(20 \mathrm{~mL})$ under argon and treated with trifluoroacetic acid $(20 \mathrm{~mL})$. The YOUNG-tube was flushed with argon and sealed. The mixture was stirred at rt. for 3-4 h. Subsequently the reaction mixture was evaporated to dryness in vacuum. The residue was dissolved in chloroform $(100 \mathrm{~mL})$ and washed with an aqueous solution of citric acid and sodium bicarbonate $(25 \mathrm{~mL}, \mathrm{pH} \approx 4)$. The aqueous phase was extracted with chloroform $(4 \times 30 \mathrm{~mL})$ and the combined organic phases were dried with magnesium sulfate. After evaporation of the solvent the crude product was obtained as colorless, viscos oils, which in high vacuum solidified. The product was used for the next step without further purification (160 mg, 40\%). ${ }^{1} \mathrm{H}$ NMR $\left(300 \mathrm{MHz}, \mathrm{CD}_{3} \mathrm{CN}\right): \delta=7.78$ $\left(\mathrm{dm},{ }^{3} J_{\mathrm{H}, \mathrm{H}}=9.1, \operatorname{ArH}, 2 \mathrm{H}\right), 7.29(\mathrm{~m}, \operatorname{ArH}, \operatorname{ArH}, \operatorname{ArH}, 5 \mathrm{H}), 7.04\left(\mathrm{dm},{ }^{3} J_{\mathrm{H}, \mathrm{H}}=9.0 \mathrm{ArH}\right.$, $2 \mathrm{H}$ ), $4.75\left(\mathrm{dm},{ }^{2} J_{\mathrm{H}, \mathrm{F}}=47.7 \mathrm{~Hz}, \mathrm{CH}_{2} \mathrm{~F}, 2 \mathrm{H}\right), 4.65\left(\mathrm{dd},{ }^{3} J_{\mathrm{H}, \mathrm{H}}=8.5,{ }^{3} J_{\mathrm{H}, \mathrm{H}}=6.0, \mathrm{NCH}, 1 \mathrm{H}\right)$, $4.57\left(\mathrm{~d},{ }^{2} J_{\mathrm{H}, \mathrm{H}}=16.1, \mathrm{NCH}_{2}, \mathrm{H}_{\mathrm{A}}, 1 \mathrm{H}\right), 4.52\left(\mathrm{dd},{ }^{3} J_{\mathrm{H}, \mathrm{F}}=17.6,{ }^{2} J_{\mathrm{H}, \mathrm{H}}=3.0, \mathrm{CF}=\mathrm{CH}_{2}, \mathrm{H}_{c i s}\right.$, 
$1 \mathrm{H}), 4.32\left(\mathrm{~d},{ }^{2} J_{\mathrm{H}, \mathrm{H}}=16.1, \mathrm{NCH}_{2}, \mathrm{H}_{\mathrm{B}}, 1 \mathrm{H}\right), 4.30\left(\mathrm{dm},{ }^{3} J_{\mathrm{H}, \mathrm{F}}=29.4 \mathrm{~Hz}, \mathrm{CH}_{2} \mathrm{CH}_{2} \mathrm{~F}, 2 \mathrm{H}\right)$, $4.14\left(\mathrm{dd},{ }^{3} J_{\mathrm{H}, \mathrm{F}}=51.8,{ }^{2} J_{\mathrm{H}, \mathrm{H}}=3.4, \mathrm{CF}=\mathrm{CH}_{2}, \mathrm{H}_{\text {trans }}, 1 \mathrm{H}\right), 2.76\left(\mathrm{ddd},{ }^{3} J_{\mathrm{H}, \mathrm{F}}=14.7,{ }^{2} J_{\mathrm{H}, \mathrm{H}}=\right.$ 13.7, $\left.{ }^{3} J_{\mathrm{H}, \mathrm{H}}=6.0, \mathrm{NCH}_{2}, \mathrm{H}_{\mathrm{A}}, 1 \mathrm{H}\right), 2.47\left(\mathrm{ddd},{ }^{3} J_{\mathrm{H}, \mathrm{F}}=22.2,{ }^{2} J_{\mathrm{H}, \mathrm{H}}=15.4,{ }^{3} J_{\mathrm{H}, \mathrm{H}}=8.5, \mathrm{NCH}_{2}\right.$, $\left.\mathrm{H}_{\mathrm{B}}, 1 \mathrm{H}\right) .{ }^{13} \mathrm{C}$ NMR $\left(75 \mathrm{MHz}, \mathrm{CD}_{3} \mathrm{CN}\right): \delta=171.2(\mathrm{CO}), 163.2\left(\mathrm{~d},{ }^{1} J_{\mathrm{C}, \mathrm{F}}=254.7 \mathrm{~Hz}, \mathrm{CF}\right)$, 163.1 ( $\mathrm{ArCO}), 138.2\left(\mathrm{ArCCH}_{2}\right), 133.0\left(\mathrm{ArCSO}_{2}\right), 131.0(\mathrm{ArCH}), 129.6(\mathrm{ArCH}), 129.3$ (ArCH), $128.7(\mathrm{ArCH}), 115.7(\mathrm{ArCH}), 93.8\left(\mathrm{~d},{ }^{2} J_{\mathrm{C}, \mathrm{F}}=18.9 \mathrm{~Hz}, \mathrm{CF}=\mathrm{CH}_{2}\right), 83.1\left(\mathrm{~d},{ }^{1} J_{\mathrm{C}, \mathrm{F}}=\right.$ $\left.167.5 \mathrm{~Hz}, \mathrm{CH}_{2} \mathrm{~F}\right), 68.8\left(\mathrm{~d},{ }^{2} J_{\mathrm{C}, \mathrm{F}}=19.2 \mathrm{~Hz}, \mathrm{CH}_{2} \mathrm{CH}_{2} \mathrm{~F}\right), 57.9(\mathrm{NCH}), 50.8\left(\mathrm{NCH}_{2}\right), 34.2$ $\left(\mathrm{d},{ }^{2} J_{\mathrm{C}, \mathrm{F}}=28.0 \mathrm{~Hz}, \mathrm{NCHCH}_{2}\right) .{ }^{19} \mathrm{~F}$ NMR $\left(282 \mathrm{MHz}, \mathrm{CD}_{3} \mathrm{CN}\right): \delta=-97.7$ (dddd, ${ }^{3} J_{\mathrm{H}, \mathrm{F}}=$ $\left.50.9 \mathrm{~Hz},{ }^{3} J_{\mathrm{H}, \mathrm{F}}=22.1 \mathrm{~Hz},{ }^{3} J_{\mathrm{H}, \mathrm{F}}=17.7 \mathrm{~Hz},{ }^{3} J_{\mathrm{H}, \mathrm{F}}=13.3 \mathrm{~Hz}, \mathrm{CF}\right),-224.0\left(\mathrm{tt},{ }^{2} J_{\mathrm{H}, \mathrm{F}}=47.7 \mathrm{~Hz}\right.$, $\left.{ }^{3} J_{\mathrm{H}, \mathrm{F}}=29.5 \mathrm{~Hz}, \quad \mathrm{CH}_{2} \mathrm{~F}\right)$. MS-ES(+)-EM: $m / z=448.1009\left[(\mathrm{M}+\mathrm{Na})^{+}\right]$calcd. For $\mathrm{C}_{20} \mathrm{H}_{31} \mathrm{~F}_{2} \mathrm{NO}_{5} \mathrm{SNa}^{+}$: 448.1006 .

(2S)-2-[N-Benzyl-4-(2-fluoroethoxy)phenylsulfonamido]-4-fluoro-N-[(tetrahydro-2H-pyran-2-yl)oxy]pent-4-enamide ((S)-8a)

To a solution of (S)-2-\{[N-benzyl-4-(2-fluoroethoxy)phenyl]sulfonamido\}-4-fluoropen$\mathrm{t}$-4-enoic acid ((S)-7a) (100 mg, $0.235 \mathrm{mmol})$ in DMF $(0.06 \mathrm{mmol} / \mathrm{mL}, 1 \mathrm{~mL})$ 1-hydroxybenzotriazole hydrate (HOBT, 1.2 eq., $38 \mathrm{mg}, \quad 0.282 \mathrm{mmol}$ ), 4-methylmorpholine (NMM, 3.0 eq., $78 \mu \mathrm{L}, 249.70 \mathrm{mmol}$ ), O-tetrahydro-2- $\mathrm{H}$-pyran-2-yl-hydroxylamine (3.1 eq., $85 \mathrm{mg}, 0.729 \mathrm{mmol}$ and $N$-[(dimethylamino)-propyl $]-N$ '-ethylcarbodiimide hydrochloride (EDC, 1.4 eq., $63 \mathrm{mg}, 0.329 \mathrm{mmol}$ ) were added. After stirring overnight at room temperature the reaction mixture was diluted with water $(20 \mathrm{~mL})$ and extracted with ethyl acetate $(3 \times 5 \mathrm{~mL})$. The combined organic phases were washed successively with water, $5 \%$ aqueous $\mathrm{KHSO}_{4}$, saturated aqueous $\mathrm{NaHCO}_{3}$ and brine, and dried over magnesium sulfate. After removing the solvent under reduced pressure column chromatographic purification (silica gel, CH/EA 2:1) yielded the THP-protected hydroxamic acid as a light brown wax (92 mg, 75\%). ${ }^{1} \mathrm{H}$ $\operatorname{NMR}\left(300 \mathrm{MHz}, \mathrm{CDCl}_{3}\right): \delta=9.19(\mathrm{~s}, \mathrm{NH}, 1 \mathrm{H}), 9.15(\mathrm{~s}, \mathrm{NH}, 1 \mathrm{H}), 7.77\left(\mathrm{~d},{ }^{3} J_{\mathrm{H}, \mathrm{H}}=8.9 \mathrm{~Hz}\right.$, $\operatorname{ArH}, 2 \mathrm{H}), 7.72\left(\mathrm{~d},{ }^{3} J_{\mathrm{H}, \mathrm{H}}=9.0 \mathrm{~Hz}, \operatorname{Ar} H, 2 \mathrm{H}\right), 7.39-7.21(\mathrm{~m}, \operatorname{Ar} H, 5 \mathrm{H}), 6.98\left(\mathrm{~d},{ }^{3} J_{\mathrm{H}, \mathrm{H}}=\right.$ $9.0 \mathrm{~Hz}, \operatorname{ArH}, 2 \mathrm{H}), 6.97\left(\mathrm{~d},{ }^{3} J_{\mathrm{H}, \mathrm{H}}=9.0 \mathrm{~Hz}, \operatorname{ArH}, 2 \mathrm{H}\right), 4.92-4.88(\mathrm{~m}, \mathrm{NHOCHO}), 4.79$ $\left(\mathrm{dm},{ }^{2} J_{\mathrm{H}, \mathrm{F}}=47.4 \mathrm{~Hz}, \mathrm{CH}_{2} \mathrm{~F}, 2 \mathrm{H}\right), 4.66\left(\mathrm{AB}, \mathrm{d},{ }^{2} J_{\mathrm{H}, \mathrm{H}}=16.0 \mathrm{~Hz}, \mathrm{NCH}_{2}, 1 \mathrm{H}\right), 4.58-4.45(\mathrm{~m}$, $\left.\mathrm{NCH}, \mathrm{NCH}_{2}, 2 \mathrm{H}\right), 4.43\left(\mathrm{dd}, \mathrm{CFCH}_{2}, \mathrm{H}_{\text {cis }},{ }^{3} J_{\mathrm{H}, \mathrm{F}}=17.1 \mathrm{~Hz},{ }^{2} J_{\mathrm{H}, \mathrm{F}}=3.0 \mathrm{~Hz}, 1 \mathrm{H}\right), 4.39(\mathrm{dd}$, $\left.\mathrm{CFCH}_{2}, \mathrm{H}_{c i s},{ }^{3} J_{\mathrm{H}, \mathrm{F}}=17.1 \mathrm{~Hz},{ }^{2} J_{\mathrm{H}, \mathrm{F}}=3.0 \mathrm{~Hz}, 1 \mathrm{H}\right), 4.27\left(\mathrm{dm},{ }^{3} J_{\mathrm{H}, \mathrm{F}}=27.7 \mathrm{~Hz}, \mathrm{O}-\mathrm{CH}_{2} \mathrm{CH}_{2} \mathrm{~F}\right.$, $2 \mathrm{H}), 4.10\left(\mathrm{dd}, \mathrm{CFCH}_{2}, \mathrm{H}_{\text {trans }},{ }^{3} \mathrm{~J}_{\mathrm{H}, \mathrm{F}}=49.9 \mathrm{~Hz},{ }^{2} \mathrm{~J}_{\mathrm{H}, \mathrm{F}}=3.1 \mathrm{~Hz}, 1 \mathrm{H}\right), 3.95-3.84(\mathrm{~m}, \mathrm{NHO}-$ $\left.\mathrm{CHOCH}_{2}, 1 \mathrm{H}\right), 3.70-3.55\left(\mathrm{~m}, \mathrm{NHOCHOCH}_{2}, 1 \mathrm{H}\right), 2.93-2.58\left(\mathrm{AB}, \mathrm{m}, \mathrm{NCHCH}_{2}, 1 \mathrm{H}\right)$, 2.47-2.18 (A, m, $\left.\mathrm{NCHCH}_{2}, 1 \mathrm{H}\right), 1.88-1.50$ (m, THP-CH, $\left.6 \mathrm{H}\right) .{ }^{13} \mathrm{C}$ NMR $(75 \mathrm{MHz}$, $\left.\mathrm{CDCl}_{3}\right): \delta=165.8(\mathrm{CONH}), 165.6(\mathrm{CONH}), 162.0\left(\mathrm{qArCOCH} \mathrm{CH}_{2} \mathrm{~F}\right), 161.2\left(\mathrm{~d},{ }^{1} J_{\mathrm{C}, \mathrm{F}}=\right.$ $\left.256.3 \mathrm{~Hz}, \mathrm{CFCH}_{2}\right), 161.2\left(\mathrm{~d},{ }^{1} J_{\mathrm{C}, \mathrm{F}}=256.0 \mathrm{~Hz}, \mathrm{CFCH}_{2}\right), 136.6\left(\mathrm{qArCCH} \mathrm{Ar}_{2} \mathrm{~N}\right), 136.5$ $\left(\mathrm{qArCCH} \mathrm{H}_{2} \mathrm{~N}\right), 131.5(\mathrm{qArCSO}), 129.7(\mathrm{ArCH}), 129.7(\mathrm{ArCH}), 128.7(\mathrm{ArCH}), 128.7$ $(\mathrm{ArCH}), 128.5(\mathrm{ArCH}), 128.4(\mathrm{ArCH}), 127.8(\mathrm{ArCH}), 127.8(\mathrm{ArCH}), 114.7(\mathrm{ArCH})$, 114.7 ( $\mathrm{ArCH}), 102.1$ ( $\mathrm{NHOCHO}), 101.7(\mathrm{NHOCHO}), 93.7$ (d, ${ }^{2} J_{\mathrm{C}, \mathrm{F}}=19.0 \mathrm{~Hz}, \mathrm{CFCH}_{2}$ ), $93.6\left(\mathrm{~d},{ }^{2} J_{\mathrm{C}, \mathrm{F}}=18.9 \mathrm{~Hz}, \mathrm{CFCH}\right), 81.4\left({ }^{1} J_{\mathrm{C}, \mathrm{F}}=171.6 \mathrm{~Hz}, \mathrm{OCH}_{2} \mathrm{CH}_{2} \mathrm{~F}\right), 67.4\left(\mathrm{~d},{ }^{2} J_{\mathrm{C}, \mathrm{F}}=\right.$ $\left.20.5 \mathrm{~Hz}, \mathrm{OCH}_{2} \mathrm{CH}_{2} \mathrm{~F}\right), 62.0\left(\mathrm{NHOCHOCH}_{2}\right), 54.7(\mathrm{NCH}), 54.5(\mathrm{NCH}), 48.6\left(\mathrm{NCH}_{2}\right)$, $48.5\left(\mathrm{NCH}_{2}\right), \quad 32.3 \quad\left(\mathrm{~d},{ }^{2} J_{\mathrm{C}, \mathrm{F}}=27.6 \mathrm{~Hz}, \quad \mathrm{NCHCH}_{2} \mathrm{CF}\right), 32.0 \quad\left(\mathrm{~d},{ }^{2} J_{\mathrm{C}, \mathrm{F}}=26.9 \mathrm{~Hz}\right.$,

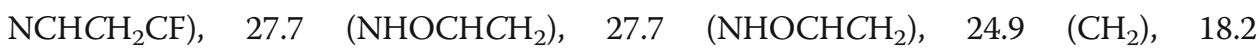
$\left(\mathrm{NHOCHCH}_{2} \mathrm{CH}_{2}\right) .{ }^{19} \mathrm{~F}$ NMR $\left(282 \mathrm{MHz}, \mathrm{CD}_{3} \mathrm{CN}\right): \delta=-97.47\left(\mathrm{~m}, \mathrm{CFCH}_{2}, 1 \mathrm{~F}\right),-97.72$ 
(m, $\left.\mathrm{CFCH}_{2}, 1 \mathrm{~F}\right),-223.89\left(\mathrm{tt},{ }^{2} J_{\mathrm{H}, \mathrm{F}}=47.3,{ }^{3} J_{\mathrm{H}, \mathrm{F}}=27.6 \mathrm{~Hz}, \mathrm{OCH}_{2} \mathrm{CH}_{2} \mathrm{~F}, 1 \mathrm{~F}\right),-223.91(\mathrm{tt}$, ${ }^{2} J_{\mathrm{H}, \mathrm{F}}=47.3,{ }^{3} J_{\mathrm{H}, \mathrm{F}}=27.6 \mathrm{~Hz}, \mathrm{OCH}_{2} \mathrm{CH}_{2} \mathrm{~F}, 1 \mathrm{~F}$ ) (two signal sets due to the presence of two stereoisomers). MS-ES-EM: $m / z=547.1683\left[(\mathrm{M}+\mathrm{Na})^{+}\right]$calcd for $\mathrm{C}_{25} \mathrm{H}_{30} \mathrm{~F}_{2} \mathrm{~N}_{2} \mathrm{O}_{6} \mathrm{SNa}^{+}$: 547.1685 .

(S)-2-[N-Benzyl-4-(2-fluoroethoxy)phenylsulfonamido]-4-fluoro-N-hydroxypent-4-enamide ((S)-9a) To a solution of (2S)-2-[N-benzyl-4-(2-fluoroethoxy)phenylsulfonamido]-4-fluoro- $N$-[(tetrahydro-2H-pyran-2-yl)oxy]pent-4-enamide ((S)-8a) $(92 \mathrm{mg}, 0.175 \mathrm{mmol})$ in 1,4-dioxane $(0.5 \mathrm{~mL} / 0.18 \mathrm{mmol}) 4 \mathrm{~N}$ hydrochloric acid in 1,4-dioxane (4 eq.) and methanol $(0.5 \mathrm{~mL} /$ $0.18 \mathrm{mmol}$ ) were added and the reaction mixture was stirred at room temperature. The reaction progress was monitored by TLC (ethyl acetate). After complete conversion the reaction mixture was diluted with ethyl acetate $(20 \mathrm{~mL})$, washed with water $(3 \times 10 \mathrm{~mL})$ and dried $\left(\mathrm{MgSO}_{4}\right)$. The solvent was removed under reduced pressure and the product was purified by column chromatography (silica gel, CH/EA 2:1). The product was obtained as a light brown wax (33 mg, 43\%). ${ }^{1} \mathrm{H}$ NMR (400 MHz, $\mathrm{CDCl}_{3}$ ): $\delta=9.19$ (s, $\left.1 \mathrm{OH}\right), 7.72$ $\left(\mathrm{d},{ }^{3} J_{\mathrm{H}, \mathrm{H}}=8.9 \mathrm{~Hz}, \operatorname{ArH}, 2 \mathrm{H}\right), 7.35-7.25(\mathrm{~m}, \mathrm{ArH}, 5 \mathrm{H}), 6.97\left(\mathrm{~d},{ }^{3} J_{\mathrm{H}, \mathrm{H}}=8.9 \mathrm{~Hz}, \operatorname{ArH}, 2 \mathrm{H}\right), 4.79$ $\left(\mathrm{dm},{ }^{2} J_{\mathrm{H}, \mathrm{F}}=47.3 \mathrm{~Hz}, \mathrm{CH}_{2} \mathrm{~F}, 2 \mathrm{H}\right), 4.54\left(\underline{\mathrm{AB}}, \mathrm{d},{ }^{2} J_{\mathrm{H}, \mathrm{H}}=15.6 \mathrm{~Hz}, \mathrm{NCH}_{2}, 1 \mathrm{H}\right), 4.52(\mathrm{~m}, \mathrm{NCH}$, $1 \mathrm{H}), 4.46\left(\mathrm{AB}, \mathrm{d},{ }^{2} J_{\mathrm{H}, \mathrm{H}}=15.7 \mathrm{~Hz}, \mathrm{NCH}_{2}, 1 \mathrm{H}\right), 4.38\left(\mathrm{dd}, \mathrm{CFCH}_{2}, \mathrm{H}_{c i s},{ }^{3} J_{\mathrm{H}, \mathrm{F}}=17.0 \mathrm{~Hz},{ }^{2} J_{\mathrm{H}, \mathrm{F}}=\right.$ $2.9 \mathrm{~Hz}, 1 \mathrm{H}), 4.26\left(\mathrm{dm},{ }^{3} J_{\mathrm{H}, \mathrm{F}}=27.6 \mathrm{~Hz}, \mathrm{CH}_{2} \mathrm{FCH}_{2}, 2 \mathrm{H}\right), 4.08\left(\mathrm{dd}, \mathrm{CFCH}_{2}, \mathrm{H}_{\text {trans }},{ }^{3} J_{\mathrm{H}, \mathrm{F}}=\right.$ $\left.49.7 \mathrm{~Hz},{ }^{2} J_{\mathrm{H}, \mathrm{F}}=3.1 \mathrm{~Hz}, 1 \mathrm{H}\right), 2.85-2.69$ (AB, m, $\left.\mathrm{NCHCH}_{2}, 1 \mathrm{H}\right), 2.38$ (Aㅛ, m, NCHCH ${ }^{13} \mathrm{C}$ NMR (101 MHz, $\left.\mathrm{CDCl}_{3}\right): \delta=166.4(\mathrm{CONH}), 162.1\left(\mathrm{qArCOCH} \mathrm{CH}_{2} \mathrm{~F}\right), 161.1\left(\mathrm{~d},{ }^{1} J_{\mathrm{C}, \mathrm{F}}\right.$ $\left.=256.3 \mathrm{~Hz}, \mathrm{CFCH}_{2}\right), 136.1\left(\mathrm{qArCCH} \mathrm{A}_{2} \mathrm{~N}\right), 131.3\left(\mathrm{qArCSO}_{2}\right), 129.7(\mathrm{ArCH}), 128.6(\mathrm{ArCH})$, 128.6 (ArCH), $128.0(\mathrm{ArCH}), 114.8(\mathrm{ArCH}), 93.8\left(\mathrm{~d},{ }^{2} J_{\mathrm{C}, \mathrm{F}}=18.9 \mathrm{~Hz}, \mathrm{CFCH}_{2}\right), 81.4\left(\mathrm{~d},{ }^{1} J_{\mathrm{C}, \mathrm{F}}\right.$ $\left.=171.7 \mathrm{~Hz}, \mathrm{OCH}_{2} \mathrm{CH}_{2} \mathrm{~F}\right), 67.4\left(\mathrm{~d},{ }^{2} J_{\mathrm{C}, \mathrm{F}}=20.4 \mathrm{~Hz}, \mathrm{OCH}_{2} \mathrm{CH}_{2} \mathrm{~F}\right), 54.2(\mathrm{NCH}), 48.7\left(\mathrm{NCH}_{2}\right)$, $31.7\left(\mathrm{~d},{ }^{2} J_{\mathrm{C}, \mathrm{F}}=27.7 \mathrm{~Hz}, \mathrm{NCHCH}_{2} \mathrm{CF}\right) .{ }^{19} \mathrm{~F} \mathrm{NMR}\left(282 \mathrm{MHz}, \mathrm{CDCl}_{3}\right): \delta=-97.58\left(\mathrm{~m}, \mathrm{CFCH}_{2}\right.$, 1F), - 223.84 (tt, $\left.{ }^{2} J_{\mathrm{H}, \mathrm{F}}=47.3,{ }^{3} J_{\mathrm{H}, \mathrm{F}}=27.6 \mathrm{~Hz}, \mathrm{OCH}_{2} \mathrm{CH}_{2} \mathrm{~F}, 1 \mathrm{~F}\right) . \mathrm{MS}-\mathrm{ES}-\mathrm{EM}: m / z=463.1120$ $\left[(\mathrm{M}+\mathrm{Na})^{+}\right]$calcd for $\mathrm{C}_{20} \mathrm{H}_{22} \mathrm{~F}_{2} \mathrm{~N}_{2} \mathrm{O}_{5} \mathrm{SNa}^{+}$: 463.1110. HPLC $\mathrm{t}_{R}=9.05 \mathrm{~min}(100 \%)$.

\section{Radiochemistry}

\section{General methods}

Radiofluorinations were carried out on a modified PET tracer radiosynthesizer (TRACERLab FxFDG, GE Healthcare). The recorded data were processed by the TRACERLab Fx software (GE Healthcare). Separation and purification of the radiosynthesized compounds were performed on the following semi-preparative radio-HPLC system $\mathbf{B}$ $(\lambda=254 \mathrm{~nm}$ ): K-500 and K-501 pump, K-2000 UV detector (Herbert Knauer GmbH), $\mathrm{NaI}(\mathrm{TI})$ Scintibloc 51 SP51 $\gamma$-detector (Crismatec) and a Nucleosil 100-10 C18 column $(5 \mu, 250 \times 8 \mathrm{~mm})$. The recorded data were processed by the GINA Star software (Raytest Isotopenmessgeräte $\mathrm{GmbH})$. The HPLC method B1 started with a linear gradient from $30 \%$ to $70 \% \mathrm{CH}_{3} \mathrm{CN}$ in water (0.1\% TFA) over $30 \mathrm{~min}$, holding for $5 \mathrm{~min}$, followed by a linear gradient from $70 \%$ to $30 \% \mathrm{CH}_{3} \mathrm{CN}$ in water (0.1\% TFA) over $5 \mathrm{~min}$, with a flow rate of $4 \mathrm{~mL} \cdot \mathrm{min}^{-1}$. Radiochemical purities and specific activities were determined using the analytical radio-HPLC system $\mathbf{C}$ and HPLC method C1. HPLC System C: Two Smartline 1000 pumps and a Smartline UV detector 2500 (Herbert Knauer $\mathrm{GmbH}$ ), a GabiStar $\gamma$-detector (Raytest Isotopenmessgeräte $\mathrm{GmbH}$ ) and a Nucleosil 100-5 C-18 column $(250 \mathrm{~mm} \times 4.6 \mathrm{~mm})$. The recorded data were processed by the 
GINA Star software (Raytest Isotopenmessgeräte GmbH). HPLC method C1 started isocratic with $40 \% \mathrm{CH}_{3} \mathrm{CN}$ in water $(0.1 \%$ TFA) for $22 \mathrm{~min}$, followed by a linear gradient from $40 \%$ to $90 \% \mathrm{CH}_{3} \mathrm{CN}$ in water $(0.1 \%$ TFA) over $4 \mathrm{~min}$, followed by a linear gradient from $90 \%$ to $40 \% \mathrm{CH}_{3} \mathrm{CN}$ in water $(0.1 \%$ TFA) over $4 \mathrm{~min}$, with a flow rate of $1 \mathrm{~mL} \cdot \mathrm{min}^{-1}$. No-carrier-added aqueous $\left[{ }^{18} \mathrm{~F}\right]$ fluoride was produced on a RDS $111 \mathrm{e}$ cyclotron (CTI-Siemens) by irradiation of a $1.2 \mathrm{~mL}$ water target using $10 \mathrm{MeV}$ proton beams on $97.0 \%$ enriched $\left[{ }^{18} \mathrm{O}\right]$ water by the ${ }^{18} \mathrm{O}(\mathrm{p}, \mathrm{n}){ }^{18} \mathrm{~F}$ nuclear reaction.

(S)-2-[N-Benzyl-4-(2-[ ${ }^{18}$ F]fluoroethoxy)phenylsulfonamido]-4-fluoro-N-hydroxypent-4-enamide ((S)- $\left.\left[{ }^{18} F\right] 9 a\right)$

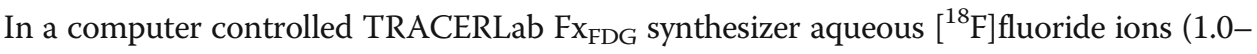
5.0 GBq) from the cyclotron target were passed through an anion exchange resin (SepPak Light Waters Accell Plus QMA cartridge, preconditioned with $5 \mathrm{~mL}$ of $1 \mathrm{M} \mathrm{K}_{2} \mathrm{CO}_{3}$ and $10 \mathrm{~mL}$ of water for injection). $\left[{ }^{18} \mathrm{~F}\right]$ Fluoride ions were eluted from the resin with a mixture of $40 \mu \mathrm{L}$ of $1 \mathrm{M} \mathrm{K}_{2} \mathrm{CO}_{3}, 200 \mu \mathrm{L}$ of water for injection, and $800 \mu \mathrm{L}$ of DNA-grade $\mathrm{CH}_{3} \mathrm{CN}$ containing $18 \mathrm{mg}(48 \mu \mathrm{mol})$ of Kryptofix2.2.2 $\left(\mathrm{K}_{222}\right)$ in the reactor. Subsequently, the aqueous $\mathrm{K}\left(\mathrm{K}_{222}\right)\left[{ }^{18} \mathrm{~F}\right] \mathrm{F}$ solution was carefully evaporated to dryness in vacuo. An amount of $\sim 5.0 \mathrm{mg}(\sim 8.4 \mu \mathrm{mol})$ of compound $(S)-9 \mathbf{b}$ and carefully dried $\mathrm{K}\left(\mathrm{K}_{222}\right)\left[{ }^{18} \mathrm{~F}\right] \mathrm{F}$ residue were heated at $84{ }^{\circ} \mathrm{C}$ in $1 \mathrm{~mL}$ DNA-grade $\mathrm{CH}_{3} \mathrm{CN}$ for $20 \mathrm{~min}$. The mixture was cooled to $40{ }^{\circ} \mathrm{C}$, diluted with $10 \mathrm{~mL}$ water for injection and passed through a Waters Sep-Pak Light C18 cartridge. The cartridge was washed with additional $10 \mathrm{~mL}$ water for injection and eluted with $0.5 \mathrm{~mL}$ warm DMF (warmed to $90{ }^{\circ} \mathrm{C}$ before elution). The eluate was diluted with $0.5 \mathrm{~mL}$ water for injection and purified by gradient-radio HPLC B using the gradient radio-HPLC method $\mathbf{B 1}$. The product fraction of compound $(S)-\left[{ }^{18} \mathrm{~F}\right] \mathbf{9 a}$ (retention time

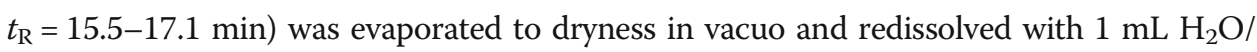
EtOH (9:1) The radiosynthesis provided $(S)-\left[{ }^{18} \mathrm{~F}\right] 9 \mathbf{a}$ with an overall radiochemical yield of $31-46 \%$ (based on cyclotron-derived $\left[{ }^{18} \mathrm{~F}\right]$ fluoride ions, $n=3$ ) in 95-110 min from end of radionuclide production. $(S)-\left[{ }^{18} \mathrm{~F}\right] 9 \mathrm{a}$ was isolated in radiochemical purities of $>97 \%$ and a molar activity in the range of $2.8-3.4 \mathrm{GBq} / \mu \mathrm{mol}$ at the end of radiosynthesis. The molar activity was determined using analytical HPLC $\mathbf{C}$ and method $\mathbf{C 1}$ (retention time of $\left.(S)-\left[{ }^{18} \mathrm{~F}\right] 9 \mathrm{a}: t_{\mathrm{R}}=16.2 \mathrm{~min}\right)$.

\section{Determination of the partition coefficient $(\log D(\exp )$.}

The lipophilicity of the radioligand $(S)-\left[{ }^{18} \mathbf{F}\right] 9 \mathbf{a}$ was assessed by determination of the water/ octanol partition coefficients following a published procedure (Prante et al. 2006). In brief, approximately $20 \mathrm{kBq}$ of $(S)-\left[{ }^{18} \mathrm{~F}\right] 9 \mathrm{a}$ were mixed with equal amounts $(0.5 \mathrm{~mL})$ of PBS ( $\mathrm{pH}$ 7.4) and 1-octanol and the resulting biphasic system was mixed vigorously for $1 \mathrm{~min}$ at rt. The tubes were centrifuged (3000 rpm, $2 \mathrm{~min}$ ) and $400 \mu \mathrm{L}$ of the octanol phase were separated and again mixed with equal amounts $(400 \mu \mathrm{L})$ of PBS. The resulting biphasic system was again mixed vigorously of $1 \mathrm{~min}$ at rt. After centrifugation (3000 rpm, $2 \mathrm{~min}$ ) three samples of $100 \mu \mathrm{L}$ of each layer were counted in a gamma counter (Wallac Wizard, Perkin-Elmer Life Science). The partition coefficient was determined by calculating the ratio $\mathrm{cpm}(\mathrm{octanol}) / \mathrm{cpm}(\mathrm{PBS})$ and expressed as $\log D($ exp. $)\left(\log \left(\mathrm{cpm}_{\mathrm{octanol}} / \mathrm{cpm}_{\mathrm{PBS}}\right)\right)$. Two independent experiments were performed in triplicate and data were provided as mean values \pm standard deviation. 
In vitro enzyme inhibition assays (Table 1 )

The inhibition potencies of (R)-9a and (S)-9a against activated MMP-2 and -9 were assayed using the synthetic fluorometric substrate (7-methoxycoumarin-4-yl)acetyl-Pro-Leu-GlyLeu-(3-(2,4-dinitrophenyl)-L-2,3-diaminopropionyl)Ala-Arg- $\mathrm{NH}_{2}$ (R\&D Systems) as described previously (Huang et al. 1997). Briefly, MMP-2 and -9 (each at $2 \mathrm{nM}$ ) and test compounds at varying concentrations $(10 \mathrm{pM}$ to $1 \mathrm{mM})$ in Tris $(50 \mathrm{mM}), \mathrm{pH} 7.5$, containing $\mathrm{NaCl}(0.2 \mathrm{M}), \mathrm{CaCl}_{2}(5 \mathrm{mM}), \mathrm{ZnSO}_{4}(20 \mu \mathrm{M})$, and $0.05 \%$ Brij 35 were preincubated at $37^{\circ} \mathrm{C}$ for $30 \mathrm{~min}$. An aliquot of substrate ( $10 \mu \mathrm{L}$ of a $50 \mu \mathrm{M}$ solution) was added to the enzyme inhibitor mixture $(90 \mu \mathrm{L})$, and the fluorescence changes were monitored using a TriStar2 Multimode Reader LB 942, (Berthold) with excitation and emission wavelengths of 340 and $405 \mathrm{~nm}$, respectively. Reaction rates were measured from the initial $10 \mathrm{~min}$ and plotted as a function of inhibitor concentration. From the resulting inhibition curves, the $\mathrm{IC}_{50}$ values were calculated by nonlinear regression analysis using the Grace 5.1.8 software (Linux).

\section{Biostability and metabolism study}

Approximately 11.1 MBq $(S)-\left[{ }^{18} \mathrm{~F}\right]$ 9a (in a maximum volume of $200 \mu \mathrm{L}$ ) was injected into three mice each via tail vein injection. The animals were sacrificed at $30 \mathrm{~min}$ p.i.. Whole blood was obtained, weighed, and centrifuged at $3000 \mathrm{rpm}$ ( $3 \mathrm{~min}$ ) to isolate plasma. Urine was also collected. The muscle, brain, kidneys, and liver were harvested and homogenized in lysis buffer (1\% SDS in PBS buffer). An aliquot of each sample $(400 \mu \mathrm{L})$ was subsequently removed, mixed with $400 \mu \mathrm{L}$ of acetonitrile and $100 \mu \mathrm{L}$ of $3 \%$ acetic acid in acetonitrile, vigorously mixed, and placed on dry ice for $3 \mathrm{~min}$. After thawing, the samples were centrifuged at $13000 \mathrm{rpm}(8 \mathrm{~min})$ to allow for the separation of supernatant from the pellet. The supernatant was then removed and assayed for radioactivity in a PerkinElmer Wizard $\gamma$-counter $(20 \mathrm{~s})$. The samples were analyzed by HPLC, using a $\gamma$-detector (Raytest GmbH/Agilent). HPLC was done on a Phenomenex C18 column $(250 \mathrm{~mm} \times 4.6 \mathrm{~mm})$ using a gradient method with acetonitrile and water (both having $0.05 \%$ TFA).

\section{Animals}

Adult C57/BL6 mice (male, 21-23 g) were anesthetized by isoflurane $/ \mathrm{O}_{2}$, and one lateral tail vein was cannulated using a $27 \mathrm{Ga}$ needle connected to $15 \mathrm{~cm}$ polyethylene catheter tubing. $(S)-\left[{ }^{18} \mathrm{~F}\right] 9 \mathrm{a}(500 \mathrm{kBq} / \mathrm{g}$ bodyweight) was injected as a bolus $(50 \mu \mathrm{L}$ of compound flushed with $100 \mu \mathrm{L}$ of saline) via the tail vein, and subsequent PET scanning was performed.

\section{Small animal PET scanning}

PET experiments were carried out using a sub-millimeter high resolution $(0.7 \mathrm{~mm}$ full width at half maximum) small animal scanner (32 module quadHIDAC, Oxford Positron Systems Ltd., Oxford, UK) with uniform spatial resolution $(<1 \mathrm{~mm})$ over a large cylindrical field (165 mm diameter, $280 \mathrm{~mm}$ axial length) (Schäfers et al. 2005).

List-mode data were acquired for $120 \mathrm{~min}$ and reconstructed into dynamic time frames using an iterative reconstruction algorithm. Subsequently, the scanning bed was transferred to the computed tomography (CT) scanner (Inveon, Siemens Medical Solutions, U.S.) and a CT acquisition with a spatial resolution of $80 \mu \mathrm{m}$ was performed for 
each mouse. Reconstructed image data sets were co-registered based on extrinsic markers attached to the multimodal scanning bed and the image analysis software (Inveon Research Workplace 3.0, Siemens Medical Solutions, USA). Three-dimensional volumes of interest (VOIs) were defined over the respective organs in CT data sets, transferred to the co-registered PET data and analyzed quantitatively. Regional uptake was calculated as percentage of injected dose by dividing counts per milliliter in the VOI by total counts in the mouse multiplied by $100(\% \mathrm{ID} / \mathrm{mL})$. Routes of tracer elimination were assessed by VOI segmentation of kidneys and bladder (renal route), and liver, gall bladder, small and large intestine (hepatobiliary route). Total activity for each route was calculated as percentage of injected dose by dividing the total counts in the respective VOI by total counts in the mouse multiplied by 100 (\%ID).

\section{Results and discussion}

Previous attempts to radiofluorinate analogs of CGS 27030A (see Fig. 1) resulted in low yields and inadmissible long synthesis times. Neither direct radiofluorination of the 3-pyridylmethyl aromatic core (Wagner et al. 2009) nor fluoroalkylation of the phenol HO-CGS 27030A in either a two- or three-step procedure using 2- $\left[{ }^{18} \mathrm{~F}\right]$ fluoro-1-tosyloxyethane led to sufficient radiochemical yields in appropriate radiosynthesis times. In the first example, high reaction temperatures led to decomposition of the molecule and in the second example unavoidable protection and subsequent deprotection of the hydroxamic acid function resulted in prolonged radiosynthesis times (Breyholz et al. 2007). In contrast, the radiosynthesis of an analog of the CGS 25966 derivative (see Table 1) was accomplished in a one-step procedure via direct nucleophilic substitution of its tosylate precursor in good radiochemical yields (RCY: 45.6 $\pm 5.6 \%$ ) and radiosynthesis times of $110 \pm 10 \mathrm{~min}$ from the end of radionuclide production (Wagner et al. 2007). Furthermore, a GMP compliant fully automated radiosynthesis of the CGS 25966 derivative (RCY $=14.9 \pm 3.9 \%$ in $57 \pm 2 \mathrm{~min}$ ) was prepared to enable first-in-man studies (Wagner et al. 2011).

CGS derivatives (R)-CGS 25966 and (R)-CGS 27030A show similar inhibition potencies towards MMP-2 and -9 and a substitution of the picolyl moiety by a benzyl ring does not crucially influence the binding affinities of the lead structures (Fig. 1). Both, the dissatisfying radiochemical yields for ${ }^{18}$ F-labelled CGS $27030 \mathrm{~A}$ derivatives and the similar inhibition potencies of the picolyl and benzyl substituted CGS derivatives encouraged us to (radio)synthesize a benzyl variant of our $\gamma$-fluorinated $\alpha$-sulfonylaminohydroxamic acid derivatives 1 and $\mathbf{2}$ (Fig. 1).

From the radiochemical point of view, introduction of $\left[{ }^{18} \mathrm{~F}\right]$ fluorine into the $\gamma$-position of the $\alpha$-amino acid is difficult. Additionally, all attempts to replace leaving groups such as tosyl or mesyl by fluoride to prepare ${ }^{18} \mathrm{~F}$-labelled compound $\mathbf{1}$, failed due to an obvious cyclization reaction (Behrends et al. 2015). Therefore, a second fluorine atom has to be introduced into the core structure analog to the radiolabeling procedure of the CGS derivative $\left[{ }^{18} \mathrm{~F}\right]$ FEtO-CGS 25966 bearing a 2-fluoroethoxy instead of the methoxy group in $p$-position of the benzene sulfonamide group. This can be realized in a one-step procedure starting from the tosylate precursor (Wagner et al. 2011). Previous studies have shown that the $p$-fluoroethoxy motif at the benzene sulfonamide group is well tolerated without affecting the MMP inhibition potency (Hugenberg et al. 2013).

To evaluate the MMP potency of the benzyl substituted $\gamma$-fluorinated $\alpha$-sulfonylaminohydroxamic acid derivative, both enantiomeric forms of the nonradioactive 
fluorinated target compound were synthesized and tested by in vitro fluorometric MMP assays.

\section{Chemistry}

The syntheses of the $\gamma$-fluorinated $\alpha$-sulfonylaminohydroxamic acid derivatives and the precursor for radiolabeling are depicted in Scheme 1. Building blocks for the synthesis of the MMPIs are represented by the $p$-fluoroethoxy and $p$-tosylethoxyphenylsulfonyl chlorides $\mathbf{3 a}$ and $\mathbf{3 b}$ as well as by the enantiomerically enriched $\gamma$-fluorinated amino acid tert-butyl esters $(R)-\mathbf{4}$ and (S)-4. $p$-(2-Fluoroethoxy)phenylsulfonyl chloride (3a) and 2-[4-(chlorosulfonyl)phenoxy]ethyl-4-methylbenzenesulfonate (3b) were prepared according to literature procedure (Wagner et al. 2011). $\gamma$-Fluorinated $\alpha$-amino acid tert-butyl esters $(R)-\mathbf{4}$ and $(S)-\mathbf{4}$ were synthesized by diastereoselective alkylation of Schiff's bases, derived from $(+)-(R, R, R)-2$-hydroxy-3-pinanone and glycine tert-butyl ester with 3-bromo-2-fluoropropene following a literature procedure (Laue et al. 2000). Substitution at their $\mathrm{N}$-terminal position yielded the sulfonamides 5. $\mathrm{N}$-Alkylation with benzyl bromide gave carboxylic acid esters 6. Deprotection of the fluoroethoxy substituted carboxylic acid esters $(R)-\mathbf{6 a}$ and $(S)$-6a were realized using trifluoroacetic acid in dichloromethane at room temperature. Compound $\mathbf{6 b}$ was deprotected differently under milder conditions using Montmorillonite KSF clay (Yadav et al. 2002) in acetonitrile under reflux. Conversion of 7 into the corresponding hydroxamic acid esters 8 were achieved by O-THP hydroxylamine, EDC, HOBT, and NMM in DMF.

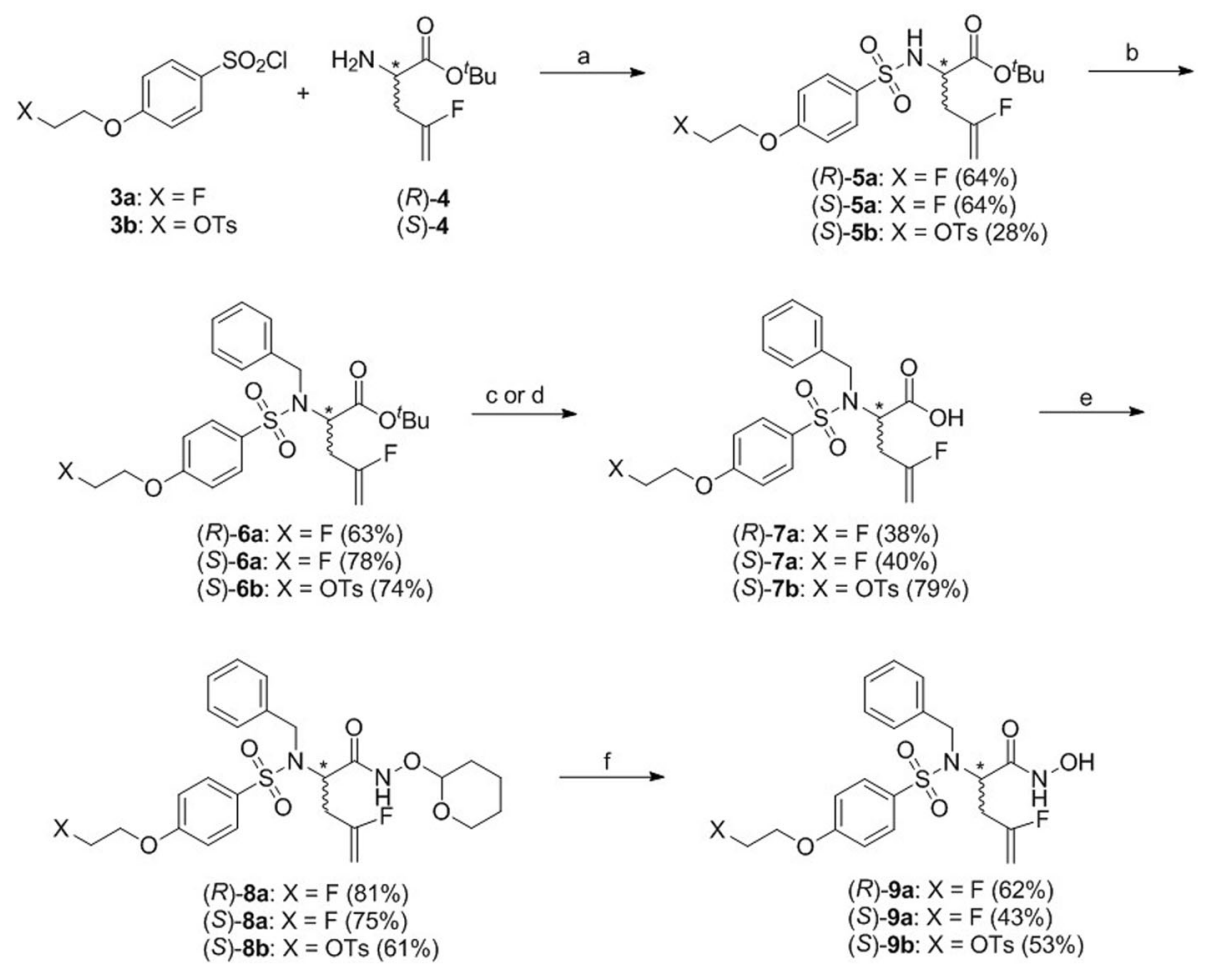

Scheme 1 Syntheses of the MMPIs and precursor 9 : Reaction conditions: (a) pyridine $0{ }^{\circ} \mathrm{C}$ - rt.; (b) $\mathrm{BnBr}$, $\mathrm{K}_{2} \mathrm{CO}_{3}$, DMF, rt.; (c) for $\mathrm{X}=\mathrm{F}$ : $\mathrm{CF}_{3} \mathrm{CO}_{2} \mathrm{H}, \mathrm{CH}_{2} \mathrm{Cl}_{2}$, rt:; (d) for $\mathrm{X}=\mathrm{OTs}$ : $\mathrm{KSF}$ clay, $\mathrm{CH}_{3} \mathrm{CN}$, reflux; (e) O-THP hydroxylamine, EDC, NMM, HOBT, DMF, rt.; (f) $4 \mathrm{~N} \mathrm{HCl}$ in dioxane/MeOH 1:1, rt. 
<smiles>[R]Oc1ccc(S(=O)(=O)N(Cc2ccccc2)C(C(=O)NO)C(C)C)cc1</smiles>

(R)-CGS 27023A (X = N, R = $\left.\mathrm{CH}_{3}\right)$ $(R)$-CGS $25966\left(\mathrm{X}=\mathrm{CH}, \mathrm{R}=\mathrm{CH}_{3}\right)$ (R)-FEtO-CGS $25966\left(\mathrm{X}=\mathrm{CH}, \mathrm{R}=\mathrm{CH}_{2} \mathrm{CH}_{2} \mathrm{~F}\right)$<smiles>[R]Oc1ccc(S(=O)(=O)N(Cc2ccccc2)C(CC(=C)F)C(=O)NO)cc1</smiles>

$(R) /(\mathrm{S})-2\left(\mathrm{X}=\mathrm{N}, \mathrm{R}=\mathrm{CH}_{3}\right)$ $(R) /(\mathrm{S})-9 \mathrm{a}\left(\mathrm{X}=\mathrm{CH}, \mathrm{R}=\mathrm{CH}_{2} \mathrm{CH}_{2} \mathrm{~F}\right)$

Fig. 2 CGS lead structures and $\gamma$-fluorinated a-sulfonylaminohydroxamic acids based MMP inhibitors 2 and $9 a$

Cleavage of the THP protecting group was performed with hydrochloric acid in a dioxane/methanol mixture, yielding the $\gamma$-fluorinated hydroxamic acids 9 (Hugenberg et al. 2012).

\section{In vitro assays}

The MMP inhibition potencies of the hydroxamic acids (R)-9a and (S)-9a (Fig. 2) against MMP-2 and -9 were measured by fluorometric in vitro inhibition assays, following the previously described procedure (Huang et al. 1997). The resulting $\mathrm{IC}_{50}$ values of the $\gamma$-fluorinated hydroxamic acids are displayed in Table 2 and were compared to those of the parent compounds $(R)-\mathbf{1}$ and $(S)-\mathbf{1}$ and the CGS lead compounds. With $\mathrm{IC}_{50}$ values of 10.4 $\mathrm{nM}$ (MMP-2) and $0.5 \mathrm{nM}$ (MMP-9) for (R)-9a and $0.3 \mathrm{nM}$ (MMP-2) and 0.1 nM (MMP-9) for (S)-9a the investigated MMP inhibitors show even higher inhibition potencies against MMP-2 and -9 compared to $(R)-\mathbf{1}$ and $(S)-\mathbf{1}$. In contrast to the CGS lead compounds, where the $(R)$-enantiomers are significantly more

Table 2 MMP inhibition potencies and $\operatorname{cog} D(\log D)$ values of lead structures and target novel hydroxamic acids

\begin{tabular}{|c|c|c|c|c|c|c|c|}
\hline \multirow[b]{2}{*}{ Compound } & \multicolumn{4}{|l|}{$\mathrm{IC}_{50}[\mathrm{nM}]^{\mathrm{a}}$} & \multicolumn{3}{|c|}{$\log D$ values } \\
\hline & $\overline{\mathrm{R}}$ & $X$ & MMP-2 & MMP-9 & $\overline{\log D^{b}}$ & $\log D($ exp.) & Ref. \\
\hline$\overline{(R)-C G S ~ 27023 A}$ & $\mathrm{CH}_{3}$ & $\mathrm{~N}$ & $20^{c}$ & $8^{c}$ & 1.49 & & $\begin{array}{l}\text { (Mac Pherson } \\
\text { et al., 1997) }\end{array}$ \\
\hline (R)-CGS 25966 & $\mathrm{CH}_{3}$ & $\mathrm{CH}$ & $11^{\mathrm{c}}$ & $27^{c}$ & 2.72 & & $\begin{array}{l}\text { (Scozzafava } \\
\text { and Supuran, } \\
\text { 2000) }\end{array}$ \\
\hline (R)-FEtO- CGS 25966 & $\mathrm{CH}_{2} \mathrm{CH}_{2} \mathrm{~F}$ & $\mathrm{CH}$ & $4 \pm 3$ & $50 \pm 27$ & 2.92 & $2.02 \pm 0.03$ & $\begin{array}{l}\text { (Wagner et al., } \\
\text { 2011) }\end{array}$ \\
\hline$(R)-2$ & $\mathrm{CH}_{3}$ & $N$ & $9.3 \pm 2.0$ & $8.3 \pm 0.1$ & 0.81 & & $\begin{array}{l}\text { (Behrends et } \\
\text { al., 2015) }\end{array}$ \\
\hline (S)-2 & $\mathrm{CH}_{3}$ & $N$ & $7.2 \pm 0.1$ & $4.9 \pm 0.4$ & 0.81 & & $\begin{array}{l}\text { (Behrends et } \\
\text { al., 2015) }\end{array}$ \\
\hline$(R)-\mathbf{9 a}$ & $\mathrm{CH}_{2} \mathrm{CH}_{2} \mathrm{~F}$ & $\mathrm{CH}$ & $10.4 \pm 5.9$ & $0.5 \pm 0.3$ & 2.23 & & \\
\hline (S)-9a & $\mathrm{CH}_{2} \mathrm{CH}_{2} \mathrm{~F}$ & $\mathrm{CH}$ & $0.3 \pm 0.03$ & $0.1 \pm 0.01$ & 2.23 & $1.16 \pm 0.2^{d}$ & \\
\hline
\end{tabular}

${ }^{a}$ Standard deviations based on three independent experiments are given for $\mathrm{IC}_{50}$ values in $\mathrm{nM}$ range

${ }^{\mathrm{b}} \mathrm{c} \log D$ values were calculated by ChemAxon $\log D$ predictor, $(\log D=\log P$ at physiological $\mathrm{pH} 7.4)$

${ }^{c} \mathrm{~K}_{\mathrm{i}}$ values, where SDs are not denoted

${ }^{\mathrm{d}} \log D$ value was determined for compound $(S)-\left[{ }^{18} \mathrm{~F}\right] 9 \mathbf{a}$ 
active, (S)-9a is more potent than its $(R)$-enantiomer, which was also observed for the $\gamma$-fluorinated $\alpha$-sulfonylaminohydroxamic acid $(S)$-1.

Obviously, the substitution of the picolyl moiety by a benzyl ring and the replacement of the methoxy group in $p$-position of the benzene sulfonyl unit by a fluoroethyl group did not diminish the inhibition potencies.

Table 2 also displays the calculated $\log D$ values $(\log D)$ of the modified $\gamma$-fluorinated hydroxamic acids to indicate the changes of the lipophilicities caused by the structural modifications. Compared to FEtO-CGS25966 $(\log D=2.92)$, the $\gamma$-fluorinated hydroxamic acids are slightly more hydrophilic. However, in comparison to the lead structure CGS27023A the calculated $\log D$ values indicate a shift towards higher lipophilicity. Additionally, the $\log D$ value of the radiofluorinated analogue $(S)-\left[{ }^{18} \mathrm{~F}\right] \mathbf{9 a}(\log D((S)-9 \mathbf{a})$ $=1.16)$ was experimentally determined. Likewise to the lead structure $\left[{ }^{18} \mathrm{~F}\right]$ FEtO-CGS25966 the $\log D(\exp )$ of $(S)-\left[{ }^{18} \mathrm{~F}\right] \mathbf{9 a}$ differs from the calculated $\log D$ $(\operatorname{cog} D((S)-9 \mathbf{a})=2.23)$ by around 1 unit. In comparison to $\left[{ }^{18} \mathrm{~F}\right] \mathrm{FEtO}-\mathrm{CGS} 25966$ the $\gamma$-fluorinated hydroxamic acid $(S)-\left[{ }^{18} \mathrm{~F}\right] 9 \mathrm{a}$ is 7 times more hydrophilic, which might be caused by the fluoroallyl group.

The investigated MMP inhibitors $(R)$ - and (S)-9a showed excellent MMP inhibition potencies in the nano- and subnanomolar range and similar lipophilicities similar to the lead compounds. Despite the modifications at the sulfonamide and the substitution of the picolyl unit for benzyl, the $\gamma$-fluorinated hydroxamic acid $(S)$-enantiomer is as potent as the $(R)$-enantiomer.

\section{Radiochemistry}

The results mentioned above encouraged us to radiosynthesize the first ${ }^{18} \mathrm{~F}$-labelled $(S)$-configured $\gamma$-fluorinated MMP inhibitor based on $\alpha$-sulfonylaminohydroxamic acid to further evaluate its pharmacokinetic behavior, metabolic stability and its applicability as an in vivo MMP radiotracer (Scheme 2).

Radiosynthesis of the ${ }^{18} \mathrm{~F}$-labelled counterpart $(S)-\left[{ }^{18} \mathrm{~F}\right] \mathbf{9 a}$ was accomplished via direct nucleophilic substitution reaction of the tosylate precursor $(S)-9 \mathbf{b}$ by $\left[{ }^{18} \mathrm{~F}\right]$ fluoride according to the synthesis described for $\left[{ }^{18} \mathrm{~F}\right] \mathrm{FEtO}-\mathrm{CGS} 25966$ (Wagner et al. 2011). The radiosynthesis provided $(S)-\left[{ }^{18} \mathrm{~F}\right] 9 \mathrm{a}$ with radiochemical yields of $31-46 \%(n=3)$, radiochemical purities of $>97 \%$ in 95-110 min from the end of radionuclide production and molar activities of $2.8-3.4 \mathrm{GBq} / \mu \mathrm{mol}$ after radiosynthesis. The experimental $\log D$ value

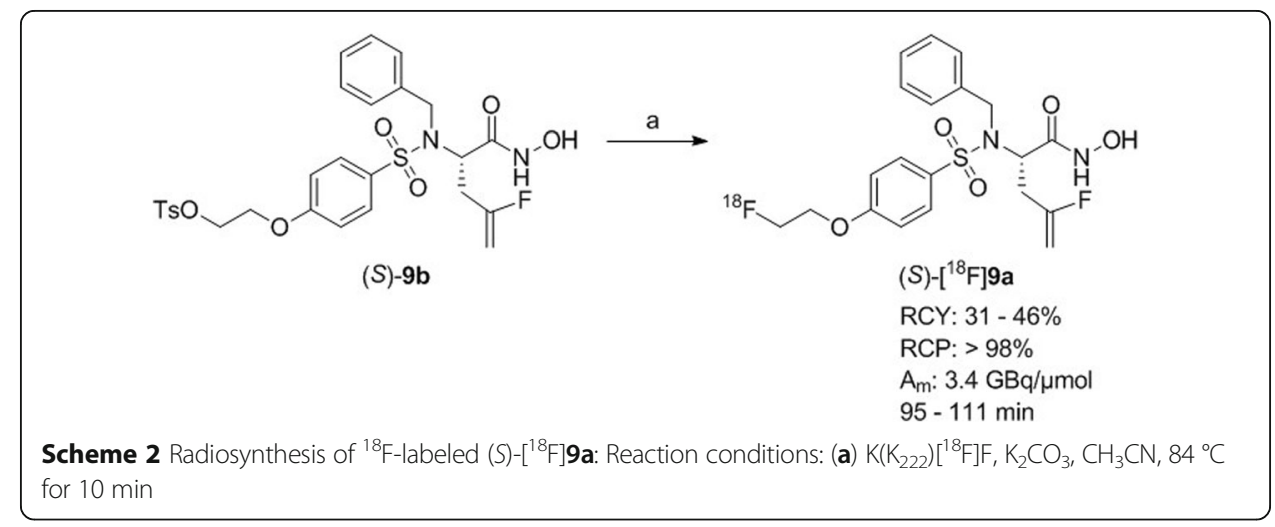


of the radioligand was determined in phosphate-buffered saline (PBS) as already described (Prante et al. 2006).

\section{In vivo biodistribution and metabolism study}

Maximum intensity projections (MIPs) of the whole body in vivo biodistribution of tracer-associated radioactivity in an adult C57/Bl6 mouse 0-2, 2-5, 5-10, 20-30 and $80-90$ min after intravenous injection of $(S)-\left[{ }^{18} \mathrm{~F}\right] 9 \mathrm{a}$ are shown in Fig. 3.

Overall, $(S)-\left[{ }^{18} \mathrm{~F}\right] \mathbf{9 a}$ is cleared fast and efficiently from the body through hepatic and renal elimination with no significant tracer remaining in non-excretion organs 80-90 min p.i (Fig. 4). Immediately upon injection of $(S)-\left[{ }^{18} \mathrm{~F}\right] 9$ a high levels of radioactivity were observed in the liver and the kidneys. While the activity in the kidney decreased ( $\mathrm{T}_{\text {max }}$ : $100 \mathrm{~s}, \mathrm{~T}_{1 / 2}: 3 \mathrm{~min}$ p.i.) in parallel to the activity in the blood, the liver first showed a further accumulation of $(S)-\left[{ }^{18} \mathrm{~F}\right] 9 \mathrm{a}$ associated activity ( $\mathrm{T}_{\max }: 3 \mathrm{~min}, \mathrm{~T}_{1 / 2}: 36 \mathrm{~min}$ p.i.) before clearance into the gallbladder and finally into the intestine (Fig. 4), representing the predominant hepatobiliary route of $(S)-\left[{ }^{18} \mathrm{~F}\right] \mathbf{9 a}$ elimination. Time-activity concentration curves of the intestinal transport of hepatobiliary eleminated $(S)-\left[{ }^{18} \mathrm{~F}\right] \mathbf{9}$ a is illustrated in Fig. S16 in the supporting information.

Defluorination of the radioligand in vivo potentially impairing image interpretation (indicated by bone uptake of $\left[{ }^{18} \mathrm{~F}\right]$ fluoride ions) was not observed in the entire dynamic imaging study. Furthermore, accumulation of $(S)-\left[{ }^{18} \mathrm{~F}\right] \mathbf{9 a}$ in organs/ tissues such as the brain, myocardium, lung and muscles, indicating unspecific binding, was not observed.

An in vivo biostability and metabolism study was performed in three 10-month old female OCR (CD1) wild type mice 30 min p.i. Representative radio-HPLC traces are shown in Fig. 5 . The retention time of unchanged $(S)-\left[{ }^{18} \mathrm{~F}\right]$ 9a tracer was between 9.8 and $10.8 \mathrm{~min}$. A total of five metabolites were detected, with retention times of $2.0 \mathrm{~min}$ (metabolite 1), $3.9 \mathrm{~min}$ (metabolite 2), $5.5 \mathrm{~min}$ (metabolite 3), $9.5 \mathrm{~min}$ (metabolite 4) and $11.4 \mathrm{~min}$ (metabolite 5) (Table 3).

In plasma, $(S)-\left[{ }^{18} \mathrm{~F}\right]$ 9a metabolizes to about $92 \%$ into four metabolites, three of these metabolites are more polar. Only $8 \%$ of the parent compound could be observed in plasma after $30 \mathrm{~min}$ in vivo in mice. In kidney homogenate, the parent tracer was

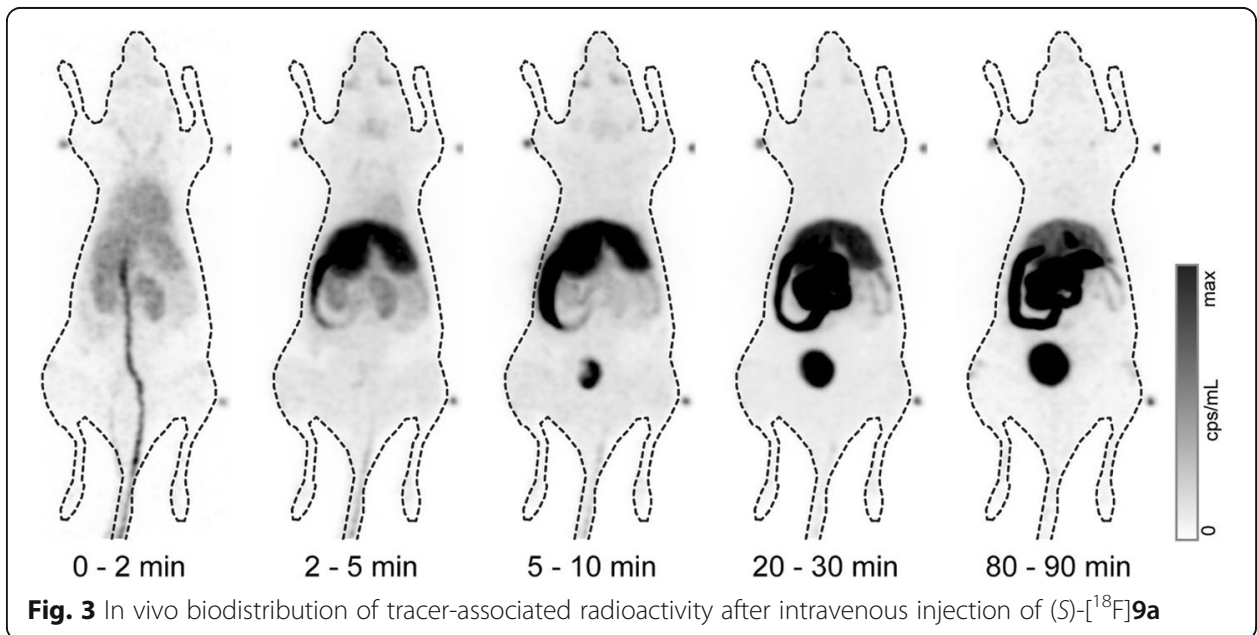




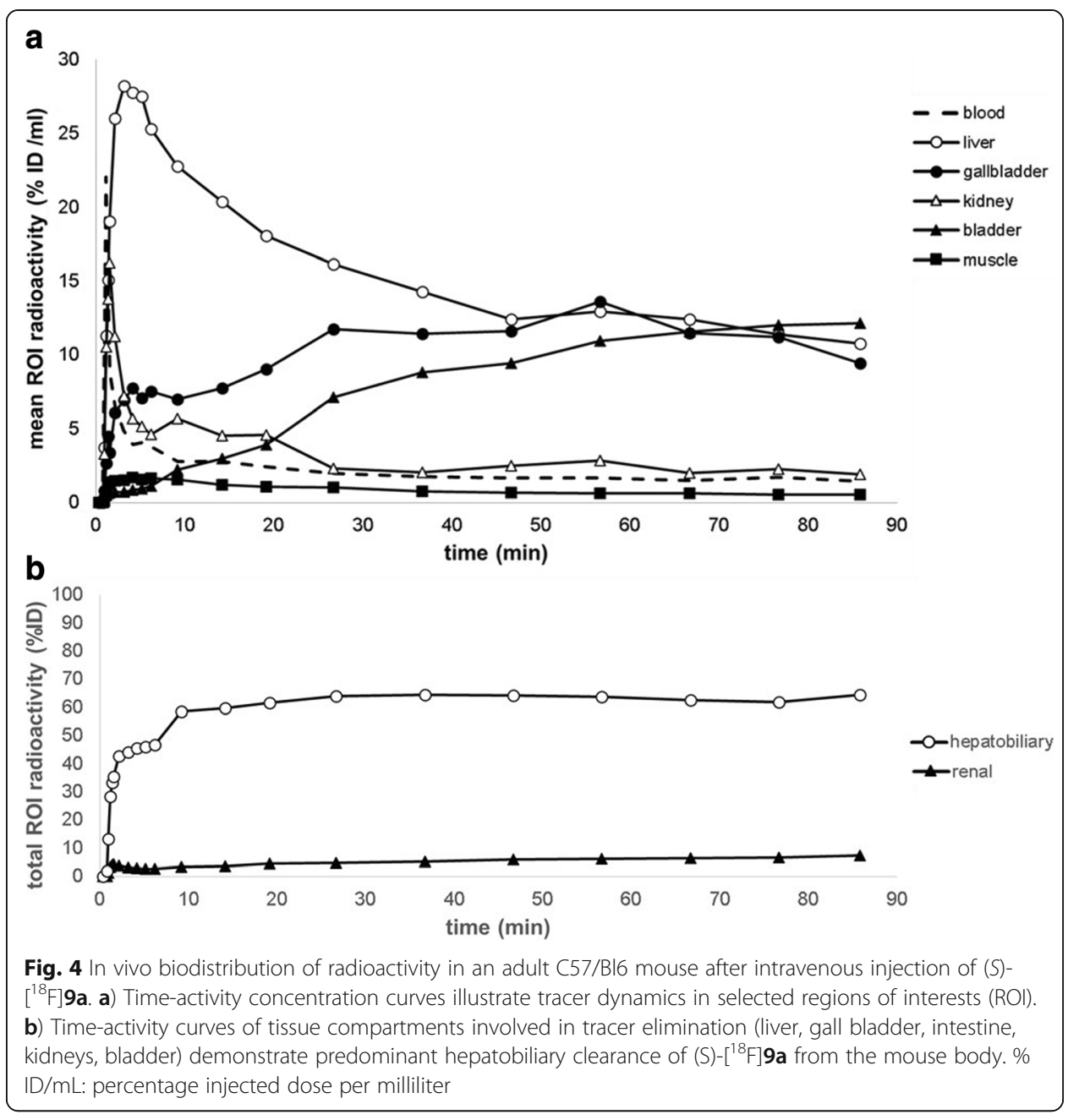

present in higher amounts, i.e. 17\% compared to the other tissues along with two polar metabolites. In urine, a complete metabolism of the parent compound to four polar metabolites was observed. Similar metabolism patterns were determined for muscle and brain $\left(17 \%\right.$ and $12 \%$ of $\left.(S)-\left[{ }^{18} \mathrm{~F}\right] \mathbf{9 a}\right)$ with only one polar metabolite formed in high amounts (83\% in muscle and $88 \%$ in brain). In all samples, except the liver, the polar metabolite 1 was formed as the main compound. In contrast, in the liver $(S)-\left[{ }^{18} \mathrm{~F}\right] \mathbf{9 a}$ showed nearly complete degradation to metabolite 4 (94\%) and only $1 \%$ of the parent compound could be detected.

Recently our group published a study about radiolabeled hydroxamate-based matrix metalloproteinase inhibitors and how chemical modifications affect their pharmacokinetics and their metabolic stability. In course of this study additionally to the in vivo metabolism study an in vitro metabolism study with mouse liver microsomes was performed. (Hugenberg et al. 2016). Polar compounds like carboxylic acids (phase I transformation) and glucuronic acid derivatives (phase II) have been identified to be the main metabolites of the modified hydroxamates during this investigation. Furthermore, analogues of CGS compounds bearing a polyethylene glycol underwent decomposition by cleavage of the ethylene glycol subunits, which was reflected by very polar metabolites. Therefore we assume that our present most polar main metabolite 1 , 


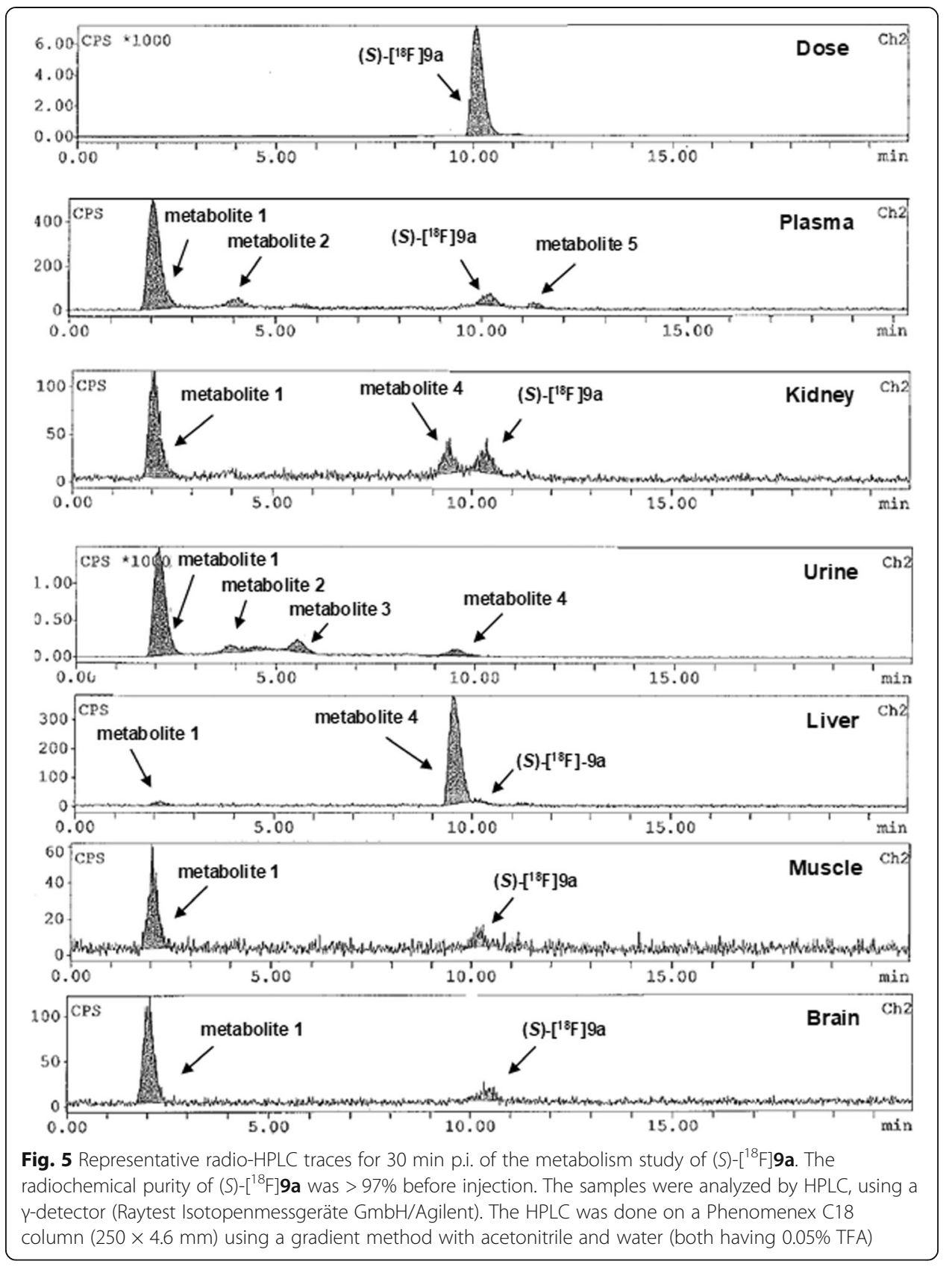

Table 3 Metabolites of $(S)-\left[{ }^{18} \mathrm{~F}\right] \mathbf{9 a}$ in plasma, kidney, urine, liver, muscle and brain

\begin{tabular}{lllllll}
\hline 30 min p.i. & \multicolumn{6}{l}{ Organ/tissue/body fluid (\% HPLC) } \\
\cline { 2 - 7 } & Plasma & Kidney & Urine & Liver & Muscle & Brain \\
\hline$(S)-\left[^{18} \mathrm{~F}\right] 9 \mathrm{a}: t_{\mathrm{R}} \approx 10.3 \mathrm{~min}$ & 8 & 17 & - & 1 & 17 & 12 \\
metabolite $1: t_{\mathrm{R}} \approx 2.0 \mathrm{~min}$ & 81 & 66 & 76 & 4 & 83 & 88 \\
metabolite $2: t_{\mathrm{R}} \approx 3.9 \mathrm{~min}$ & 6 & - & 9 & & - & - \\
metabolite $3: t_{\mathrm{R}} \approx 5.5 \mathrm{~min}$ & 2 & - & 9 & & - & - \\
metabolite $4: t_{\mathrm{R}} \approx 9.5 \mathrm{~min}$ & - & 17 & 6 & 94 & - & - \\
metabolite $5: t_{\mathrm{R}} \approx 11.4 \mathrm{~min}$ & 3 & - & - & 1 & - & - \\
\hline
\end{tabular}


might be $\left[{ }^{18} \mathrm{~F}\right]$ fluoroethanol or $\left[{ }^{18} \mathrm{~F}\right]$ fluoracetic acid. Such small molecules are able to easily enter the brain (Peana et al. 2016), what furthermore can be confirmed by the fact that metabolite 1 is found to be the main metabolite in the brain fraction.

The tracer and its metabolites are predominantly cleared through the liver and the gallbladder (92\%) and to a lower degree by renal excretion (8\%). 90 min post injection $73 \%$ of the injected tracer activity was cleared from the blood and tissues. Compared to the lead compound CGS 25966 no significant differences in the clearance characteristics were observed.

$(S)-\left[{ }^{18} \mathrm{~F}\right] 9$ a metabolizes in plasma, kidneys, urine, muscle and brain to about $90 \%$ and the polar metabolite 1 was formed as the major metabolite. In contrast, in the liver a nearly complete degradation of the parent compound to the slightly more polar metabolite 4 was observed.

\section{Conclusion}

In a six step sequence, two new enantiomerically pure MMP-2 and -9 inhibitors were synthesized in $7.7 \%$ and $6.4 \%$ overall yield, respectively. As a consequence of the presence of a fluorine atom in the amino acid core, both enantiomers are biologically almost equally active, likely attributable to additional interactions of the fluorine with the active site of the enzymes. Surprisingly, the $(S)$-enantiomer was even more active than the $(R)$-isomer in contrast to the CGS lead structures. Also, the new inhibitors are more hydrophilic (experimental $\log D=1.2$ ) compared to the analog derived from CGS 25966 (experimental $\log D=2.0$ ). The most active inhibitor was ${ }^{18} \mathrm{~F}$-labelled $(S)-\left[{ }^{18} \mathrm{~F}\right] \mathbf{9 a}$ (38\% radiochemical yield, $>97 \%$ radiochemical purity, $3.4 \mathrm{GBq} / \mu \mathrm{mol}$ molar specific activity, 102 min synthesis time). Despite higher hydrophilicity, the in vivo biodistribution of $(S)-\left[{ }^{18} \mathrm{~F}\right] \mathbf{9 a}$ possess no significant differences, compared to the slightly more lipophilic ${ }^{18}$ F-fluoroethoxy substituted lead compound CGS 25966. Unfortunately, this radiotracer is metabolically not stable in wild type mice, which impairs further clinical development of this tracer. Two major radioactive metabolites were found in different concentrations in different organs. However, metabolic defluorination was not observed. Higher metabolic stability was found for CGS-based MMPIs bearing a 1,4-disubstituted 1,2,3-triazole core instead of the benzyl moiety. Therefore, metabolic stability of $(S)-\left[{ }^{18} \mathrm{~F}\right] \mathbf{9 a}$ could possibly be improved by the substitution of the benzyl ring by a triazole moiety.

\section{Additional file}

Additional file 1: (Experimental procedures, analytical data for compounds (R)-5a, (S)-5b, $(R)-\mathbf{6} \mathbf{a},(S)-\mathbf{6 b},(R)-\mathbf{7 a}$ $(S)-\mathbf{7 b},(R)-\mathbf{8} \mathbf{a},(S)-\mathbf{8 b},(R)-\mathbf{9} \mathbf{a}$ and (S)-9b , and copies of NMR spectra) associated with this article can be found at. (DOCX $4305 \mathrm{~kb})$

\footnotetext{
Abbreviations

\% ID: percentage injected dose; $\mathrm{CH}$ : Cyclohexane; EA: Ethyl acetate; EDC: $N^{\prime \prime}$-ethyl- $N^{\prime \prime}$-(3-dimethylaminopropyl)carbodiimide hydrochloride; EM: Exact mass; GMP: Good Manufacturing Practice; HOBT: 1-hydroxy-benzotriazole; ICR: Imprinting control region; $K_{222}$ : Kryptofix2.2.2; $\log D$ : $\log P$ at physiological pH (7.4); MMP: Matrix metalloproteinase; MMPI: Matrix metalloproteinase inhibitor; NMM: 4-methyl-morpholine; p.i.: Post injection; PET: Positron emission tomography; RCP: Radiochemical product; RCY: Radiochemical yield (decay corrected); ROI: Region of interest; SD: Standard deviation; SPECT: Single photon emission computed tomography; THP: Tetrahydropyranyl; TIMP: Tissue inhibitors of metalloproteinase; $t_{\mathrm{R}}$ : Retention time
} 


\section{Acknowledgements}

The authors thank Christine Bätza, Stefanie Bouma, Claudia Essmann, Sven Fatum, Wiebke Gottschlich, Sandra Höppner, Marlena Kattenbeck, Sarah Köster, Nina Kreienkamp, Sandra Laumann, Roman Priebe and Katrin Reckmann for technical support, Umesh B. Gangadharmath, Changhui Liu and Vani P. Morcharla of the Siemens Medical Solutions team for performing the metabolism study, and the staff members of the Organic Chemistry Institute, University of Münster, Germany, for collecting spectroscopic and analytical data.

\section{Funding}

This work was supported by the Deutsche Forschungsgemeinschaft (DFG), Collaborative Research Center 656 (Molecular Cardiovascular Imaging), Projects B1, A2, Z05 and C06) University of Münster, and the Interdisciplinary Center of Clinical Research (IZKF core unit SmAP), Münster, Germany.

\section{Availability of data and materials}

All data generated or analysed during this study are included in this published article and its Additional file 1.

\section{Authors' contributions}

$\mathrm{VH}$ participated in the design of the study, did the majority of the chemical experiments, designed and composed most of the draft manuscript and participated in its finalization. MB synthesized the precursors and core structures used for the preparation of the target molecules. SW designed and executed the radiochemical experiments, participated in writing the corresponding part of the manuscript. SH designed and executed the biodistribution experiments, participated in writing the corresponding part of the manuscript. MS initiated the investigations on MMPIs and its application for PET imaging. HK participated in the design of the metabolism study. KS participated in the design of the metabolism study. JCW contributed to the metabolism study and to the finalization of the manuscript. LFG contributed to the metabolism study. KK was involved in the initial investigations of fluorinated CGSbased MMPIs and application in PET imaging. GH conceived and coordinated the present study, supervised the chemical syntheses, participated in the design of the manuscript and finalized it. All authors read and approved the final manuscript.

\section{Ethics approval}

All animal experiments were performed in accordance with the legal requirements of the European Community (Directive 2010/63/EU) and the corresponding German Animal Welfare Law (TierSchG, TierSchVersV) and were approved by the local authorizing agency (State Office for Nature, Environment and Consumer Protection North Rhine-Westphalia).

\section{Consent for publication}

Not applicable.

\section{Competing interests}

The authors declare that they have no competing interests.

\section{Publisher's Note}

Springer Nature remains neutral with regard to jurisdictional claims in published maps and institutional affiliations.

\section{Author details}

${ }^{1}$ European Institute for Molecular Imaging, University of Münster, Waldeyerstr. 15, D-48149 Münster, Germany.

${ }^{2}$ Department of Nuclear Medicine, University Hospital Münster, Albert-Schweitzer-Campus 1, Building A1, D-48149 Münster, Germany. ${ }^{3}$ Organisch-Chemisches Institut, Westfälische Wilhelms-Universität Münster, Corrensstraße 40, D-48149 Münster, Germany. ${ }^{4}$ Cells in Motion' Cluster of Excellence, University of Münster, Waldeyerstr. 15, D-48149 Münster, Germany. ${ }^{5}$ Siemens Medical Solutions USA, Inc., 6140 Bristol Parkway, Culver City, California 90230, USA. ${ }^{6}$ Present Address: Institute for Radiology, Nuclear Medicine and Molecular Imaging, Heart and Diabetes Center North Rhine Westphalia, University Hospital, Ruhr University Bochum, Georgstraße 11, D-32545 Bad Oeynhausen, Germany. 7Present Address: German Cancer Research Center (dkfz), Division of Radiopharmaceutical Chemistry, Im Neuenheimer Feld 280, D-69120 Heidelberg, Germany.

Received: 29 March 2018 Accepted: 2 July 2018

Published online: 27 July 2018

\section{References}

Begué JP, Bonnet-Delpon D. Bioorganic and Medicinal chemistry of fluorine. Hoboken: Wiley; 2008.

Behrends M, Wagner S, Kopka K, Schober O, Schäfers M, Kumbhar S, Waller M, Haufe G. New matrix metalloproteinase inhibitors based on $\gamma$-fluorinated a-aminocarboxylic and a-aminohydroxamic acids. Bioorg Med Chem. 2015;23: 3809-18.

Brauer PR. MMPs-role in cardiovascular development and disease. Front Biosci. 2006;11:447-78.

Breyholz HJ, Wagner S, Levkau B, Schober O, Schäfers M, Kopka K. A ${ }^{18}$ F-radiolabeled analogue of CGS 27030A as a potential agent for assessment of matrix-metalloproteinase activity in vivo. Q J Nucl Med Mol Imaging. 2007;51:24-32.

Dimas GG, Didangelos TP, Grekas DM. Matrix gelatinases in atherosclerosis and diabetic nephropathy: progress and challenges. Curr Vasc Pharmacol. 2017;15:557-65.

Fingleton B. Matrix metalloproteinases: role in cancer and metastasis. Front Biosci. 2006;11:479-91.

Gillis EP, Eastman KJ, Hill MD, Donnelly DJ, Meanwell NA. Applications of fluorine in medicinal chemistry. J Med Chem. 2015;58:8315-25. 
Gong L, Wu D, Zou J, Chen J, Chen L, Chen Y, Ni C, Yuan H. Prognostic impact of serum and tissue MMP-9 in nonsmall cell lung cancer: a systematic review and meta-analysis. Oncotarget. 2016;7:18458-68.

Gruber BL, Sorbi D, French DL, Marchese MJ, Nuovo GJ, Kew RR, Arbeit LA. Markedly elevated serum MMP-9 (gelatinase B) levels in rheumatoid arthritis: a potentially useful laboratory marker. Clin Immunol Immunop. 1996;78:161-71.

Huang W, Meng Q, Suzuki K, Nagase H, Brew K. Mutational study of the amino-terminal domain and human tissue inhibitor of metalloproteinases 1 (TIMP-1) locates an inhibitory region for matrix metallo proteinases. J Biol Chem. 1997;272:22086-91.

Huchet QA, Kuhn B, Wagner B, Kratochwil NA, Fischer H, Kansy M, Zimmerli D, Carreira EM, Müller K. Fluorination patterning: a study of structural motifs that impact physicochemical properties of relevance to drug discovery. J Med Chem. 2015;58:9041-60

Hugenberg V, Breyholz HJ, Riemann B, Hermann S, Schober O, Schäfers M, Gangadharmath U, Mocharla V, Kolb H, Walsh J, Zhang W, Kopka K, Wagner S. A new class of highly potent matrix metalloproteinase inhibitors based on triazole-substituted hydroxamates: (radio)synthesis and in vitro and first in vivo evaluation. J Med Chem. 2012;55: 4714-27.

Hugenberg V, Hermann S, Galla F, Schäfers M, Wünsch B, Kolb HC, Szardenings K, Lebedev A, Walsh JC, Morcharla VP, Gangadharmath UB, Kopka K, Wagner S. Radiolabeled hydroxamate-basedmatrixmetalloproteinase inhibitors: how chemical modifications affect pharmacokinetics and metabolic stability. Nucl Med Biol. 2016;43:424-37.

Hugenberg V, Riemann B, Hermann S, Schober O, Schäfers M, Szardenings K, Lebedev A, Gangadharmath U, Kolb H, Walsh J, Zhang W, Kopka K, Wagner S. Inverse 1,2,3-triazole-1-yl-ethyl substituted hydroxamates as highly potent matrix metalloproteinase inhibitors: (radio)synthesis, in vitro and first in vivo evaluation. J Med Chem. 2013;56: 6858-70.

Jacobsen JA, Major Jourden JL, Miller MT, Cohen SM. To bind zinc and not to bind zinc: an examination of innovative approaches to improved metalloproteinase inhibition. BBA - Mol Cell Res. 2010;1803:72-94. and references cited therein

Jia H, Zhang Q, Liu F, Zhou D. Prognostic value of MMP-2 for patients with ovarian epithelial carcinoma: a systematic review and meta-analysis. Arch Gynecol Obstet. 2017;295:689-96.

Kessenbrock K, Plaks V, Werb Z. Matrix metalloproteinases: regulators of the tumor microenvironment. Cell. 2010;141:52-67.

Laue KW, Kröger S, Wegelius E, Haufe G. Stereoselective synthesis of $\gamma$-fluorinated a-amino acids using 2-hydroxy-3pinanone as an auxiliary. Eur J Org Chem. 2000:3737-43.

Levin M, Udi Y, Solomonov I, Sagi I. Next generation matrix metalloproteinase inhibitors - novel strategies bring new prospects. BBA - Molecular Cell Research. 2017;1864:1927-39.

Li M, Yang G, Xie B, Babu K, Huang C. Changes in matrix metalloproteinase-9 levels during progression of atrial fibrillation. J Int Med Res. 2014;42:224-30.

Mac Pherson LJ, Bayburt EK, Capparelli MP, Carroll BJ, Goldstein R, Justice MR, Zhu L, Hu SI, Melton RA, Freyer L, Goldberg RL, Doughty JR, Spirito S, Blancuzzi V, Wilson D, O'Byrne EM, Ganu V, Parker DT. Discovery of CGS 27023A a non-peptidic, potent, and orally active stromelysin inhibitor that blocks cartilage degradation in rabbits. J Med Chem. 1997:40:2525-32.

Matusiak N, van Waarde A, Bischoff R, Oltenfreiter R, van de Wiele C, Dierckx RAJO, Elsinga PH. Probes for non-invasive matrix metalloproteinase-targeted imaging with PET and SPECT. Curr Pharm Des. 2013;19:4647-72.

Meanwell NA. Fluorine and fluorinated motifs in the design and application of Bioisosteres for drug design. J Med Chem. 2018;000 https://doi.org/10.1021/acs.jmedchem.7b01788

O'Hagan D. Understanding organofluorine chemistry. An introduction to the C-F bond. Chem Soc Rev. 2008;37:308-19.

Peana AT, Rosas M, Porru S, Acquas E. From ethanol to salsolinol: role of ethanol metabolites in the effects of ethanol. J Exp Neurosci. 2016;10:137-46.

Prante O, Hocke C, Löber S, Hübner H, Gmeiner P, Kuwert T. Tissue distribution of radioiodinated FAUC113: assessment of a pyrazolo[1,5-a]pyridine based dopamine D4 receptor radioligand candidate. Nuklearmedizin. 2006;45:41-8.

Purser S, Moore RR, Swallow S, Gouverneur V. Fluorine in medicinal chemistry. Chem Soc Rev. 2008;37:320-30.

Rabkin SW. The role matrix metalloproteinases in the production of aortic aneurysm. Prog Mol Biol Transl Sci. 2017;147:239-65.

Rai V, Agrawal DK. The role of damage- and pathogen-associated molecular patterns in inflammation-mediated vulnerability of athereosclerotic plaques. Can J Physiol Pharmacol. 2017;95:1245-53.

Ren F, Tang R, Zhang X, Madushi WM, Luo D, Dang Y, Li Z, Wei K, Chen G. Overexpression of MMP family members functions as prognostic biomarker for breast Cancer patients: a systematic review and meta-analysis. PLoS One. 2015;10:e0135544

Schäfers KP, Reader AJ, Kriens M, Knoess C, Schober O, Schäfers M. Performance evaluation of the 32-module quadHIDAC small animal PET scanner. J Nucl Med. 2005;46:996-1004.

Scozzafava A, Supuran $C T$. Carbonic anhydrase and matrix metalloproteinase inhibitors: sulfonylated amino acid hydroxamates with MMP inhibitory properties acta as efficient inhibitors of CA isoymes I, II, IV, and Nhydroxysulfonamides inhibit both these zinc enzymes. J Med Chem. 2000;43:3677-87.

Sternlicht MD, Werb Z. How matrix metalloproteinases regulate cell behavior. Annu Rev Cell Dev Biol. 2001;17:463-516.

Wagner S, Breyholz HJ, Höltke C, Faust A, Schober O, Schäfes M, Kopka K. A new ${ }^{18}$ F-labelled derivative of the MMP inhibitor CGS 27030A for PET: radiosynthesis and initial small animal PET studies. Appl Radiat Isot. 2009;67:606-10.

Wagner S, Breyholz HJ, Law MP, Faust A, Höltke C, Schröer S, Haufe G, Levkau B, Schober O, Schäfers M, Kopka K. Novel fluorinated derivatives of the broad-spectrum MMP inhibitors N-hydroxy-2(R)-\{[(4-methoxyphenyl)sulfonyl](benzyl)and (3-picolyl)-amino\}-3-methyl-butanamide as potential tools for molecular imaging of activated MMPs with PET. J Med Chem. 2007:50:5752-64.

Wagner S, Faust A, Breyholz HJ, Schober O, Schäfers M, Kopka K. The MMP inhibitor (R)-[N-benzyl-4- $\left(2-\left[{ }^{18} \mathrm{~F}\right]-\right.$ fluoroethoxy)phenylsulfonamido-N-hydroxy-3] methylbutanamide: improved precursor synthesis and fully automated radiosynthesis. Appl Radioat Isot. 2011;69:862-8.

Whittaker M, Floyd CD, Brown P, Gearing AJH. Design and therapeutic application of matrix metalloproteinase inhibitors. Chem Rev. 1999;99:2735-76.

Woessner JF, Nagase H. Matrix metalloproteinases and TIMPs (protein profile). New York: Oxford University Press; 2000. 
Yadav JS, Reddy BVS, Rao KS, Harikishan K. Montmorillonite clay: a novel reagent for the chemoselective hydrolysis of t-butyl esters. Synlett. 2002;5:826-8.

Yamazaki T, Taguchi T, Ojima I. Unique properties of fluorine and their relevance to medicinal chemistry and chemical biology. In: Ojima I, editor. Fluorine in medicinal chemistry and chemical biology. Chichester: Wiley-Blackwell; 2009 p. 3-46.

Zhong Y, Lu YT, Sun Y, Shi ZH, Li NG, Tang YP, Duan JA. Recent opportunities in matrix metalloproteinase inhibitor drug design for cancer. Expert Opin Drug Discov. 2018;13:75-87.

\section{Submit your manuscript to a SpringerOpen ${ }^{\circ}$} journal and benefit from:

- Convenient online submission

- Rigorous peer review

- Open access: articles freely available online

- High visibility within the field

- Retaining the copyright to your article

Submit your next manuscript at $\gg$ springeropen.com 